\title{
FINITENESS OF COUSIN COHOMOLOGIES
}

\author{
TAKESI KAWASAKI
}

\begin{abstract}
The notion of the Cousin complex of a module was given by Sharp in 1969. It wasn't known whether its cohomologies are finitely generated until recently. In 2001, Dibaei and Tousi showed that the Cousin cohomologies of a finitely generated $A$-module $M$ are finitely generated if the base ring $A$ is local, has a dualizing complex, $M$ satisfies Serre's $\left(S_{2}\right)$-condition and is equidimensional. In the present article, the author improves their result. He shows that the Cousin cohomologies of $M$ are finitely generated if $A$ is universally catenary, all the formal fibers of all the localizations of $A$ are Cohen-Macaulay, the Cohen-Macaulay locus of each finitely generated $A$-algebra is open and all the localizations of $M$ are equidimensional. As a consequence of this, he gives a necessary and sufficient condition for a Noetherian ring to have an arithmetic Macaulayfication.
\end{abstract}

\section{INTRODUCTION}

Let $A$ be a Noetherian ring. The notion of Cousin complex of an $A$-module was introduced by Sharp [22] as an analogue of Hartshorne [11]. Sharp used the vanishing of its cohomologies for investigating the Cohen-Macaulay property and Serre's $\left(S_{n}\right)$-condition on modules [21]. The aim of the present article is to study non-zero Cousin cohomologies. The main theorem of this article is the following.

Theorem 1.1. Let $A$ be a Noetherian ring and $M$ a finitely generated $A$-module. Then all the cohomology modules of the Cousin complex of $M$ are finitely generated and only finitely many of them are non-zero if

(C1) A is universally catenary;

(C2) all the formal fibers of all the localizations of $A$ are Cohen-Macaulay;

(C3) the Cohen-Macaulay locus of each finitely generated A-algebra is open;

(QU) $\operatorname{dim} M_{\mathfrak{p}}=\mathrm{ht} \mathfrak{p} / \mathfrak{q}+\operatorname{dim} M_{\mathfrak{q}}$ for any pair of prime ideals $\mathfrak{p} \supset \mathfrak{q}$ in the support of $M$.

As consequences of Theorem 1.1, we obtain two applications.

Theorem 1.2. Let $A$ be a Noetherian ring of positive dimension. Then the following statements are equivalent:

(1) A has an arithmetic Macaulayfication; that is, there is an ideal $\mathfrak{b}$ of positive height such that the Rees algebra of $\mathfrak{b}$ is Cohen-Macaulay.

Received by the editors October 8, 2003 and, in revised form, September 4, 2006.

2000 Mathematics Subject Classification. Primary 13D03; Secondary 13A30, 13F40, 13H10.

Key words and phrases. Arithmetic Macaulayfication, Cohen-Macaulay ring, Cousin complex, excellent ring.

This work was supported by the Japan Society for the Promotion of Science (the Grant-in-Aid for Scientific Research (C)(2) 13640034). 
(2) A satisfies (C1)-(C3) and

(U) A has no embedded primes and

$$
\text { ht } \mathfrak{p}=\text { ht } \mathfrak{p} / \mathfrak{q}+\text { ht } \mathfrak{q}
$$

for any pair of prime ideals $\mathfrak{p} \supset \mathfrak{q}$ in $\operatorname{Spec} A$.

Theorem 1.3. A Noetherian ring is a homomorphic image of a Cohen-Macaulay ring if and only if it satisfies (C1)-(C3) and has a codimension function. In particular, an excellent ring is a homomorphic image of a Cohen-Macaulay excellent ring if it has a codimension function.

Although the author [15] established these results for local rings without the use of the Cousin complex, we can prove them for non-local rings by using Theorem 1.1.

Next we consider the assumptions of Theorem 1.1. They are necessary in a sense.

Theorem 1.4. Let $A$ be a Noetherian catenary ring. Then the following statements are equivalent:

(1) A satisfies (C1)-(C3);

(2) for any finitely generated $A$-module $M$ satisfying (QU), all the cohomology modules of the Cousin complex of $M$ are finitely generated and only finitely many of them are non-zero.

We now describe the structure of this article. In the local case, the Cousin complex is closely related to the highest local cohomology module and hence to the canonical module. To show Theorem 1.1, we need to discuss the highest local cohomology. To do this, we give a new notion, called a p-standard sequence, in Sections 2 and 3. It is a generalization of the notion of unconditioned strong $d$-sequence. Next we consider the Koszul cohomologies of the highest local cohomology. In Section 5, we prove Theorem 1.1 for local rings. In 2001, Dibaei and Tousi [5] showed that all the Cousin cohomologies of a finitely generated $A$-module $M$ are finitely generated if $A$ is local, has a dualizing complex, and $M$ is equidimensional and satisfies Serre's $\left(S_{2}\right)$-condition. However some of their assumptions are redundant. We refine their result. In Section 6, we prove Theorem 1.1 for general rings. Since Theorem 1.1 is proved for local rings, it is enough to establish an analogue of [6, Satz 1]. The key to the proof is the support of the Cousin cohomology. We show that it is small enough by using results in Sections 2-4. We find a similar phenomenon to [8, Theorem 1]. Theorems 1.2-1.4 are proved in Section 8. We need long calculations to prove Theorem 1.2. They are only variants of Theorem 3.6, Corollaries 3.7 and 3.8 of [15]. We separate them from the proof of Theorem 1.2 and give them in Section 7 .

\section{The DEFINITION AND BASIC PROPERTIES OF P-STANDARD SEQUENCES}

Let $A$ be a commutative ring and $M$ an $A$-module.

Definition 2.1. A sequence $x_{1}, \ldots, x_{d}$ in $A$ is said to be a $d$-sequence on $M$ if

$$
\left(x_{1}, \ldots, x_{i-1}\right) M: x_{i} x_{j}=\left(x_{1}, \ldots, x_{i-1}\right) M: x_{j}
$$

whenever $1 \leq i \leq j \leq d$. The sequence $x_{1}, \ldots, x_{d}$ is said to be a strong $d$-sequence on $M$ if $x_{1}^{n_{1}}, \ldots, x_{d}^{n_{d}}$ is a $d$-sequence on $M$ for any positive integers $n_{1}, \ldots, n_{d}$. The sequence $x_{1}, \ldots, x_{d}$ is said to be an unconditioned strong d-sequence on $M$ if it is a strong $d$-sequence on $M$ in any order. 
The notion of a $d$-sequence was given by Huneke [12] and that of an unconditioned strong $d$-sequence was given by Goto and Yamagishi [9].

An unconditioned strong $d$-sequence has many good properties, but its definition is so strong that we cannot use it for our purpose. We want to give a weaker notion than that of an unconditioned strong $d$-sequence.

Note that a sequence $x_{1}, \ldots, x_{d}$ is an unconditioned strong $d$-sequence on $M$ if and only if

$$
\left(x_{\lambda}^{n_{\lambda}} \mid \lambda \in \Lambda\right) M: x_{i}^{n_{i}} x_{j}^{n_{j}}=\left(x_{\lambda}^{n_{\lambda}} \mid \lambda \in \Lambda\right) M: x_{j}^{n_{j}}
$$

for any positive integers $n_{1}, \ldots, n_{d}$, any subset $\Lambda \subsetneq\{1, \ldots, d\}$ and $i, j \in\{1, \ldots, d\} \backslash$ $\Lambda$.

Definition 2.2. A sequence $x_{1}, \ldots, x_{d}$ in $A$ is said to be an unconditioned psequence on $M$ if

$$
\left(x_{\lambda}^{n_{\lambda}} \mid \lambda \in \Lambda\right) M: x_{i}^{n_{i}}=\left(x_{\lambda}^{n_{\lambda}} \mid \lambda \in \Lambda\right) M: x_{i}
$$

for any positive integers $n_{1}, \ldots, n_{d}$, any subset $\Lambda \subsetneq\{1, \ldots, d\}$ and $i \in\{1, \ldots, d\} \backslash \Lambda$.

Let $0 \leq s<d$ be an integer. The sequence $x_{1}, \ldots, x_{d}$ is said to be a p-standard sequence on $M$ of type $s$ if

$$
\left(x_{\lambda}^{n_{\lambda}} \mid \lambda \in \Lambda\right) M: x_{i}^{n_{i}} x_{j}^{n_{j}}=\left(x_{\lambda}^{n_{\lambda}} \mid \lambda \in \Lambda\right) M: x_{j}^{n_{j}}
$$

for any positive integers $n_{1}, \ldots, n_{d}$, any subset $\Lambda \subsetneq\{1, \ldots, d\}$ and $i, j \in\{1, \ldots, d\} \backslash$ $\Lambda$ such that $i \leq j$ or $s<j$. If $x_{1}, \ldots, x_{d}$ is a p-standard sequence on $M$ of type $s$ for some $0 \leq s<d$, then we simply call it a p-standard sequence on $M$.

N. T. Cuong [3, p. 481] gave the notion of an unconditioned p-sequence. Naturally a p-standard sequence is an unconditioned p-sequence. Furthermore a sequence is an unconditioned strong $d$-sequence if and only if it is a p-standard sequence of type 0 .

The following propositions were first shown by Goto and Yamagishi for unconditioned strong $d$-sequences.

Proposition 2.3. Let $x_{1}, \ldots, x_{d}$ be an unconditioned $\mathrm{p}$-sequence on $M, n_{1}, \ldots$, $n_{d}$ positive integers, $\Lambda \subsetneq\{1, \ldots, d\}$ a subset and $i \in\{1, \ldots, d\} \backslash \Lambda$. Then

$$
\left(x_{\lambda}^{n_{\lambda}} \mid \lambda \in \Lambda\right) M: x_{i}^{n_{i}}=\sum_{\Lambda^{\prime} \subset \Lambda}\left(\prod_{\lambda \in \Lambda^{\prime}} x_{\lambda}^{n_{\lambda}-1}\right)\left[\left(x_{\lambda} \mid \lambda \in \Lambda\right) M: x_{i}\right] .
$$

Here we put $\left(x_{\lambda}^{n_{\lambda}} \mid \lambda \in \Lambda\right)=(0)$ and $\prod_{\lambda \in \Lambda} x_{\lambda}^{n_{\lambda}-1}=1$ if $\Lambda=\emptyset$.

Proof. See [9, Lemma 2.2] or [14, Theorem A.1]. Although those results are stated for an unconditioned $d$-sequence, the argument works for an unconditioned psequence.

Proposition 2.4. Let $x_{1}, \ldots, x_{d}$ be a p-standard sequence on $M$ and $n_{1}, \ldots, n_{d}$, $m_{1}, \ldots, m_{d}$ positive integers. Then

$$
\begin{aligned}
\left(x_{1}^{n_{1}+m_{1}}, \ldots, x_{d}^{n_{d}+m_{d}}\right) M: x_{1}^{m_{1}} \cdots x_{d}^{m_{d}} & \\
& =\sum_{i=1}^{d}\left(x_{1}^{n_{1}}, \ldots, x_{i-1}^{n_{i-1}}, x_{i+1}^{n_{i+1}}, \ldots, x_{d}^{n_{d}}\right) M: x_{i}+\left(x_{1}^{n_{1}}, \ldots, x_{d}^{n_{d}}\right) M .
\end{aligned}
$$


Proof. We apply the proof of $\left[9\right.$, Theorem 2.3] to the reversed sequence $x_{d}, \ldots$, $x_{2}, x_{1}$.

We work by induction on $d$. If $a \in x_{1}^{n_{1}+m_{1}} M: x_{1}^{m_{1}}$, then $x_{1}^{m_{1}} a=x_{1}^{n_{1}+m_{1}} b$ where $b \in M$. Therefore

$$
a-x_{1}^{n_{1}} b \in 0 \underset{\dot{M}}{:} x_{1}^{m_{1}}=0 \underset{\dot{M}}{\dot{:}} x_{1} .
$$

Thus $x_{1}^{n_{1}+m_{1}} M: x_{1}^{m_{1}} \subset 0::_{M} x_{1}+x_{1}^{n_{1}} M$. The opposite inclusion is obvious.

Assume that $d>1$ and make the obvious inductive assumption. Let

$$
a \in\left(x_{1}^{n_{1}+m_{1}}, \ldots, x_{d}^{n_{d}+m_{d}}\right) M: x_{1}^{m_{1}} \cdots x_{d}^{m_{d}}
$$

and $x_{1}^{m_{1}} \cdots x_{d}^{m_{d}} a=x_{1}^{n_{1}+m_{1}} b+c$ where $b \in M$ and $c \in\left(x_{2}^{n_{2}+m_{2}}, \ldots, x_{d}^{n_{d}+m_{d}}\right) M$.

Then

$$
\begin{aligned}
x_{2}^{m_{2}} \cdots x_{d}^{m_{d}} a-x_{1}^{n_{1}} b \in & \left(x_{2}^{n_{2}+m_{2}}, \ldots, x_{d}^{n_{d}+m_{d}}\right) M: x_{1}^{m_{1}} \\
= & x_{2}^{n_{2}+m_{2}-1} \cdots x_{d}^{n_{d}+m_{d}-1}\left[\left(x_{2}, \ldots, x_{d}\right) M: x_{1}\right] \\
& +\sum_{\Lambda \subsetneq\{2, \ldots d\}}\left(\prod_{\lambda \in \Lambda} x_{\lambda}^{n_{\lambda}+m_{\lambda}-1}\right)\left[\left(x_{\lambda} \mid \lambda \in \Lambda\right) M: x_{1}\right]
\end{aligned}
$$

by Proposition 2.3 because $x_{1}, \ldots, x_{d}$ is an unconditioned p-sequence. If $j \in$ $\{2, \ldots, d\} \backslash \Lambda$, then

$$
\left(x_{\lambda} \mid \lambda \in \Lambda\right) M: x_{1} \subset\left(x_{\lambda} \mid \lambda \in \Lambda\right) M: x_{1} x_{j}=\left(x_{\lambda} \mid \lambda \in \Lambda\right) M: x_{j} .
$$

Hence

$$
x_{2} \cdots x_{d}\left[\left(x_{\lambda} \mid \lambda \in \Lambda\right) M: x_{1}\right] \subset\left(x_{\lambda}^{2} \mid \lambda \in \Lambda\right) M
$$

if $\Lambda \subsetneq\{2, \ldots, d\}$. Therefore

$$
\begin{aligned}
x_{2}^{m_{2}+1} \cdots x_{d}^{m_{d}+1} a-x_{1}^{n_{1}} x_{2} \cdots x_{d} b \in & x_{2}^{n_{2}+m_{2}} \cdots x_{d}^{n_{d}+m_{d}}\left[\left(x_{2}, \ldots, x_{d}\right) M: x_{1}\right] \\
& +\left(x_{2}^{n_{2}+m_{2}+1}, \ldots, x_{d}^{n_{d}+m_{d}+1}\right) M
\end{aligned}
$$

and hence

$$
\begin{aligned}
a \in & x_{2}^{n_{2}-1} \cdots x_{d}^{n_{d}-1}\left[\left(x_{2}, \ldots, x_{d}\right) M: x_{1}\right] \\
& +\left(x_{1}^{n_{1}}, x_{2}^{n_{2}+m_{2}+1}, \ldots, x_{d}^{n_{d}+m_{d}+1}\right) M: x_{2}^{m_{2}+1} \cdots x_{d}^{m_{d}+1} .
\end{aligned}
$$

Since $x_{2}, \ldots, x_{d}$ is a p-standard sequence on $M / x_{1}^{n_{1}} M$, we obtain from the inductive hypothesis

$$
\begin{aligned}
a \in & x_{2}^{n_{2}-1} \ldots x_{d}^{n_{d}-1}\left[\left(x_{2}, \ldots, x_{d}\right) M: x_{1}\right] \\
& +\sum_{i=2}^{d}\left(x_{1}^{n_{1}}, x_{2}^{n_{2}}, \ldots, x_{i-1}^{n_{i-1}}, x_{i+1}^{n_{i+1}}, \ldots, x_{d}^{n_{d}}\right) M: x_{i} \\
& +\left(x_{1}^{n_{1}}, \ldots, x_{d}^{n_{d}}\right) M \\
\subset & \sum_{i=1}^{d}\left(x_{1}^{n_{1}}, \ldots, x_{i-1}^{n_{i-1}}, x_{i+1}^{n_{i+1}}, \ldots, x_{d}^{n_{d}}\right) M: x_{i}+\left(x_{1}^{n_{1}}, \ldots, x_{d}^{n_{d}}\right) M .
\end{aligned}
$$

The opposite inclusion is obvious. 
The next proposition is a part of $\left[9\right.$, Theorem 3.9]. Let $\boldsymbol{x}=x_{1}, \ldots, x_{d}$ be a sequence in $A$. The local cohomology modules of $M$ with respect to $\boldsymbol{x}$ are the direct limits of Koszul cohomology modules; that is,

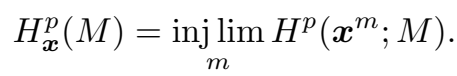

Since $K^{\bullet}(\boldsymbol{x} ; M)=K^{\bullet}\left(x_{1}, \ldots, x_{k} ; A\right) \otimes K^{\bullet}\left(x_{k+1}, \ldots, x_{d} ; M\right)$, there is a spectral sequence

$$
E_{2}^{p q}=H_{x_{1}, \ldots, x_{k}}^{p} H_{x_{k+1}, \ldots, x_{d}}^{q}(M) \Rightarrow H_{\boldsymbol{x}}^{p+q}(M) .
$$

In particular, $H_{\boldsymbol{x}}^{d}(M) \cong H_{x_{1}, \ldots, x_{k}}^{k} H_{x_{k+1}, \ldots, x_{d}}^{d-k}(M)$. If $A$ is Noetherian, then $H_{\boldsymbol{x}}^{p}(M)$ is equal to the local cohomology module of $M$ with respect to the ideal $\left(x_{1}, \ldots, x_{d}\right)$.

Proposition 2.5. Let $\boldsymbol{x}=x_{1}, \ldots, x_{d}$ be a strong d-sequence on $M$. Then

$$
H_{\boldsymbol{x}}^{p}(M)=\operatorname{inj}_{m} \lim \frac{\left(x_{1}^{m}, \ldots, x_{p}^{m}\right) M: x_{p+1}}{\left(x_{1}^{m}, \ldots, x_{p}^{m}\right) M}
$$

for $p<d$.

Proof. We work by induction on $d$. If $d=1$, then the claim is clear because $0:_{M} x_{1}^{m}=0:_{M} x_{1}$ for all $m>0$.

Assume that $d>1$ and make the obvious inductive assumption. Since there is a spectral sequence

$$
E_{2}^{p q}=H_{x_{d}}^{p} H_{x_{1}, \ldots, x_{d-1}}^{q}(M) \Rightarrow H_{\boldsymbol{x}}^{p+q}(M),
$$

we have an exact sequence

$$
0 \rightarrow H_{x_{d}}^{1} H_{x_{1}, \ldots, x_{d-1}}^{p-1}(M) \rightarrow H_{\boldsymbol{x}}^{p}(M) \rightarrow H_{x_{d}}^{0} H_{x_{1}, \ldots, x_{d-1}}^{p}(M) \rightarrow 0 .
$$

On the other hand, we also have an exact sequence

$$
\begin{aligned}
0 \rightarrow H_{x_{d}}^{0} H_{x_{1}, \ldots, x_{d-1}}^{p}(M) \rightarrow H_{x_{1}, \ldots, x_{d-1}}^{p}(M) \rightarrow & \left.\rightarrow H_{x_{1}, \ldots, x_{d-1}}^{p}(M)\right]_{x_{d}} \rightarrow H_{x_{d}}^{1} H_{x_{1}, \ldots, x_{d-1}}^{p}(M) \rightarrow 0 .
\end{aligned}
$$

If $p<d-1$, then $x_{d} H_{x_{1}, \ldots, x_{d-1}}^{p}(M)=0$ because of the inductive assumption and Remark (2) in $\left[12\right.$, p. 252]. Therefore $H_{x_{d}}^{1} H_{x_{1}, \ldots, x_{d-1}}^{p}(M)=0$ and hence

$$
\begin{aligned}
H_{\boldsymbol{x}}^{p}(M) & =H_{x_{d}}^{0} H_{x_{1}, \ldots, x_{d-1}}^{p}(M) \\
& =H_{x_{1}, \ldots, x_{d-1}}^{p}(M) \\
& =\operatorname{inj}_{m} \lim _{m} \frac{\left(x_{1}^{m}, \ldots, x_{p}^{m}\right) M: x_{p+1}}{\left(x_{1}^{m}, \ldots, x_{p}^{m}\right) M} .
\end{aligned}
$$

Since, in particular, $x_{d} H_{x_{1}, \ldots, x_{d-1}}^{d-2}(M)=0$, it follows that $H_{x_{d}}^{1} H_{x_{1}, \ldots, x_{d-1}}^{d-2}(M)=0$. Therefore

$$
\begin{aligned}
H_{\boldsymbol{x}}^{d-1}(M) & =H_{x_{d}}^{0} H_{x_{1}, \ldots, x_{d-1}}^{d-1}(M) \\
& =H_{x_{d}}^{0}\left(\operatorname{inj} \lim _{m} M /\left(x_{1}^{m}, \ldots, x_{d-1}^{m}\right) M\right) \\
& =\underset{m}{\operatorname{inj} \lim } H_{x_{d}}^{0}\left(M /\left(x_{1}^{m}, \ldots, x_{d-1}^{m}\right) M\right) \\
& =\underset{m}{\operatorname{inj} \lim } \frac{\left(x_{1}^{m}, \ldots, x_{d-1}^{m}\right) M: x_{d}}{\left(x_{1}^{m}, \ldots, x_{d-1}^{m}\right) M} .
\end{aligned}
$$




\section{The EXistence of P-Standard SEquences}

In this section, we demonstrate the ubiquity of p-standard sequences. Assume that $A$ is a Noetherian local ring with maximal ideal $\mathfrak{m}$ and $M$ a finitely generated $A$-module of dimension $d>0$.

For a finitely generated $A$-module $N$, the ideal $\mathfrak{a}(N)$ is defined to be

$$
\mathfrak{a}(N)=\prod_{0 \leq p<\operatorname{dim} N} \operatorname{ann} H_{\mathfrak{m}}^{p}(N) .
$$

Definition 3.1. Let $0 \leq s<d$ be an integer. A system of parameters $x_{1}, \ldots, x_{d}$ for $M$ is called a p-standard system of parameters for $M$ of type $s$ if

$$
x_{s+1}, \ldots, x_{d} \in \mathfrak{a}(M)
$$

and

$$
x_{i} \in \mathfrak{a}\left(M /\left(x_{i+1}, \ldots, x_{d}\right) M\right)
$$

for $i \leq s$. If $s=d-1$, then it is simply called a p-standard system of parameters for $M$.

A system of parameters $x_{1}, \ldots, x_{d}$ for $M$ is called a standard system of parameters if it satisfies the following equivalent conditions:

- the difference

$$
\ell\left(M /\left(x_{1}^{n_{1}}, \ldots, x_{d}^{n_{d}}\right) M\right)-e\left(x_{1}^{n_{1}}, \ldots, x_{d}^{n_{d}} ; M\right)
$$

is independent of the choice of positive integers $n_{1}, \ldots, n_{d}$;

- for any $p, q \geq 0$ with $p+q<d$,

$$
\left(x_{q+1}, \ldots, x_{d}\right) H_{\mathfrak{m}}^{p}\left(M /\left(x_{1}, \ldots, x_{q}\right) M\right)=0 ;
$$

- $x_{1}, \ldots, x_{d}$ is an unconditioned strong $d$-sequence.

In this case, $H_{\mathfrak{m}}^{p}(M)$ is finitely generated for $p<d$. See [20] or [25].

N. T. Cuong considered the difference (3.1.1). He showed [2] that (3.1.1) is a polynomial in $n_{1}, \ldots, n_{d}$ if and only if $x_{1}, \ldots, x_{d}$ is an unconditioned p-sequence on $M$. Next he studied the existence of such a system of parameters. He showed $[3$, p. 482] that there is a p-standard system of parameters for $M$ if $A$ has a dualizing complex and that a p-standard system of parameters is an unconditioned p-sequence. We want to show that a p-standard system of parameters is not only an unconditioned p-sequence but also a p-standard sequence.

Lemma 3.2 ([19, Theorem 3]). If $x_{1}, \ldots, x_{d}$ is a system of parameters for $M$, then

$$
\left(x_{1}, \ldots, x_{i-1}\right) M: x_{i} \subset\left(x_{1}, \ldots, x_{i-1}\right) M: \mathfrak{a}(M)
$$

for any $1 \leq i \leq d$.

Theorem 3.3. A p-standard system of parameters for $M$ of type $s$ is a p-standard sequence on $M$ of type $s$.

Proof. Let $x_{1}, \ldots, x_{d}$ be a p-standard system of parameters for $M$ of type $s$. We show the equation

$$
\left(x_{\lambda}^{n_{\lambda}} \mid \lambda \in \Lambda\right) M: x_{i}^{n_{i}} x_{j}^{n_{j}}=\left(x_{\lambda}^{n_{\lambda}} \mid \lambda \in \Lambda\right) M: x_{j}^{n_{j}}
$$

for any $n_{1}, \ldots, n_{d}>0, \Lambda \subsetneq\{1, \ldots, d\}$ and $i, j \in\{1, \ldots, d\} \backslash \Lambda$ such that $i \leq j$ or $j>s$. 
If $j>s$, then both sides of (3.3.1) are equal to

$$
\left(x_{\lambda}^{n_{\lambda}} \mid \lambda \in \Lambda\right) M: \mathfrak{a}(M)
$$

because of Lemma 3.2.

Assume that $i \leq j \leq s$ and let $p$ be the number of elements in $\{\lambda \in \Lambda \mid \lambda>$ $\left.j, n_{\lambda}>1\right\}$. We work by induction on $p$.

If $p=0$, then we work by descending induction on the number $q$ of elements in $\{\lambda \in \Lambda \mid \lambda>j\}$. If $q=d-j$, that is, $j+1, \ldots, d \in \Lambda$, then the application of Lemma 3.2 to $M /\left(x_{j+1}, \ldots, x_{d}\right) M$ shows that both sides of (3.3.1) are equal to

$$
\left(x_{\lambda}^{n_{\lambda}} \mid \lambda \in \Lambda\right) M: \mathfrak{a}\left(M /\left(x_{j+1}, \ldots, x_{d}\right) M\right) .
$$

If $q<d-j$, then there exists $k>j$ such that $k+1, \ldots, d \in \Lambda$ and $k \notin \Lambda$. If $a \in\left(x_{\lambda}^{n_{\lambda}} \mid \lambda \in \Lambda\right) M: x_{i}^{n_{i}} x_{j}^{n_{j}}$, then

$$
\begin{aligned}
a & \in\left[x_{k} M+\left(x_{\lambda}^{n_{\lambda}} \mid \lambda \in \Lambda\right) M\right]: x_{i}^{n_{i}} x_{j}^{n_{j}} \\
& =\left[x_{k} M+\left(x_{\lambda}^{n_{\lambda}} \mid \lambda \in \Lambda\right) M\right]: x_{j}^{n_{j}}
\end{aligned}
$$

because of the inductive assumption. Therefore

$$
\begin{aligned}
x_{j}^{n_{j}} a & \in\left(x_{\lambda}^{n_{\lambda}} \mid \lambda \in \Lambda\right) M: x_{i}^{n_{i}} \cap\left[\left(x_{\lambda}^{n_{\lambda}} \mid \lambda \in \Lambda\right) M+x_{k} M\right] \\
& =\left(x_{\lambda}^{n_{\lambda}} \mid \lambda \in \Lambda\right) M+\left(x_{\lambda}^{n_{\lambda}} \mid \lambda \in \Lambda\right) M: x_{i}^{n_{i}} \cap x_{k} M \\
& =\left(x_{\lambda}^{n_{\lambda}} \mid \lambda \in \Lambda\right) M+x_{k}\left[\left(x_{\lambda}^{n_{\lambda}} \mid \lambda \in \Lambda\right) M: x_{i}^{n_{i}} x_{k}\right] .
\end{aligned}
$$

It follows from Lemma 3.2 that

$$
\left(x_{\lambda}^{n_{\lambda}} \mid \lambda \in \Lambda\right) M: x_{i}^{n_{i}} x_{k}=\left(x_{\lambda}^{n_{\lambda}} \mid \lambda \in \Lambda\right) M: x_{k} .
$$

Indeed, both sides are equal to

$$
\left(x_{\lambda}^{n_{\lambda}} \mid \lambda \in \Lambda\right) M: \mathfrak{a}(M) \text { if } k>s
$$

or

$$
\left(x_{\lambda}^{n_{\lambda}} \mid \lambda \in \Lambda\right) M: \mathfrak{a}\left(M /\left(x_{k+1}, \ldots, x_{d}\right) M\right) \quad \text { if } k \leq s .
$$

Thus $x_{j}^{n_{j}} a \in\left(x_{\lambda}^{n_{\lambda}} \mid \lambda \in \Lambda\right) M$. Therefore, the proof is completed if $p=0$.

Assume that $p>0$ and make the obvious inductive assumption. We divide (3.3.1) into two parts:

$$
\begin{array}{r}
\left(x_{\lambda}^{n_{\lambda}} \mid \lambda \in \Lambda\right) M: x_{i}^{n_{i}} \subset\left(x_{\lambda}^{n_{\lambda}} \mid \lambda \in \Lambda\right) M: x_{j}, \\
\left(x_{\lambda}^{n_{\lambda}} \mid \lambda \in \Lambda\right) M: x_{j}^{n_{j}+1} \subset\left(x_{\lambda}^{n_{\lambda}} \mid \lambda \in \Lambda\right) M: x_{j} .
\end{array}
$$

Let $k \in \Lambda$ such that $k>j, n_{k}>1$ and let $\Lambda^{\prime}=\Lambda \backslash\{k\}$. If $a \in\left(x_{\lambda}^{n_{\lambda}} \mid \lambda \in\right.$ $\Lambda) M: x_{i}^{n_{i}}$, then we put $x_{i}^{n_{i}} a=x_{k}^{n_{k}} b+c$ where $b \in M, c \in\left(x_{\lambda}^{n_{\lambda}} \mid \lambda \in \Lambda^{\prime}\right) M$. The inductive assumption implies that

$$
\begin{aligned}
b & \in\left[x_{i}^{n_{i}} M+\left(x_{\lambda}^{n_{\lambda}} \mid \lambda \in \Lambda^{\prime}\right) M\right]: x_{k}^{n_{k}} \\
& =\left[x_{i}^{n_{i}} M+\left(x_{\lambda}^{n_{\lambda}} \mid \lambda \in \Lambda^{\prime}\right) M\right]: x_{k} .
\end{aligned}
$$

If we put $x_{k} b=x_{i}^{n_{i}} a^{\prime}+c^{\prime}$ where $a^{\prime} \in M, c^{\prime} \in\left(x_{\lambda}^{n_{\lambda}} \mid \lambda \in \Lambda^{\prime}\right) M$, then

$$
a^{\prime} \in\left[\left(x_{\lambda}^{n_{\lambda}} \mid \lambda \in \Lambda^{\prime}\right) M+x_{k} M\right]: x_{i}^{n_{i}}
$$

and

$$
a-x_{k}^{n_{k}-1} a^{\prime} \in\left(x_{\lambda}^{n_{\lambda}} \mid \lambda \in \Lambda^{\prime}\right) M: x_{i}^{n_{i}} .
$$

Thus

$$
a \in x_{k}^{n_{k}-1}\left[\left[\left(x_{\lambda}^{n_{\lambda}} \mid \lambda \in \Lambda^{\prime}\right) M+x_{k} M\right]: x_{i}^{n_{i}}\right]+\left(x_{\lambda}^{n_{\lambda}} \mid \lambda \in \Lambda^{\prime}\right) M: x_{i}^{n_{i}} .
$$


By using the inductive assumption twice, we find that

$$
\begin{aligned}
a & \in x_{k}^{n_{k}-1}\left[\left[\left(x_{\lambda}^{n_{\lambda}} \mid \lambda \in \Lambda^{\prime}\right) M+x_{k} M\right]: x_{i}^{n_{i}} x_{j}\right]+\left(x_{\lambda}^{n_{\lambda}} \mid \lambda \in \Lambda^{\prime}\right) M: x_{i}^{n_{i}} x_{j} \\
& \subset x_{k}^{n_{k}-1}\left[\left[\left(x_{\lambda}^{n_{\lambda}} \mid \lambda \in \Lambda^{\prime}\right) M+x_{k} M\right]: x_{j}\right]+\left(x_{\lambda}^{n_{\lambda}} \mid \lambda \in \Lambda^{\prime}\right) M: x_{j} \\
& \subset\left(x_{\lambda}^{n_{\lambda}} \mid \lambda \in \Lambda\right) M: x_{j} .
\end{aligned}
$$

Thus we obtain (3.3.2).

In the same way as above, we have

$$
\begin{aligned}
\left(x_{\lambda}^{n_{\lambda}} \mid \lambda \in \Lambda\right) M: x_{j}^{n_{j}+1} \subset & x_{k}^{n_{k}-1}\left[\left[\left(x_{\lambda}^{n_{\lambda}} \mid \lambda \in \Lambda^{\prime}\right) M+x_{k} M\right]: x_{j}^{n_{j}+1}\right] \\
& +\left(x_{\lambda}^{n_{\lambda}} \mid \lambda \in \Lambda^{\prime}\right) M: x_{j}^{n_{j}+1} .
\end{aligned}
$$

By using the inductive assumption again, we obtain (3.3.3).

The converse holds in a sense.

Proposition 3.4. Let $x_{1}, \ldots, x_{d}$ be a system of parameters for $M, 0 \leq s<d$, $n_{1} \geq 1, n_{2} \geq 2, \ldots, n_{s} \geq s$ and $n_{s+1}, \ldots, n_{d} \geq d$ integers. If $x_{1}, \ldots, x_{d}$ is a p-standard sequence on $M$ of type $s$, then $x_{1}^{n_{1}}, \ldots, x_{d}^{n_{d}}$ is a p-standard system of parameters for $M$ of type $s$.

Proof. We work by induction on $d$. If $d=1$, then $H_{\mathfrak{m}}^{0}(M)=0:_{M} x_{1}$ and hence $x_{1} \in \mathfrak{a}(M)$.

Assume that $d>1$ and make the obvious inductive assumption. Since each pstandard sequence is a strong $d$-sequence, it follows from the proof of Proposition 2.5 that $H_{\mathfrak{m}}^{p}(M)=H_{x_{1}, \ldots, x_{d}}^{p}(M)$ is annihilated by $x_{d}$ if $p<d-1$. Furthermore Proposition 2.5 itself implies $x_{d} H_{\mathfrak{m}}^{d-1}(M)=0$. Therefore $x_{d}^{n_{d}} \in \mathfrak{a}(M)$. If $s<i<d$, then $x_{1}, \ldots, x_{i-1}, x_{i+1}, \ldots, x_{d}, x_{i}$ is also a p-standard sequence on $M$ of type $s$. Therefore $x_{s+1}^{n_{s+1}}, \ldots, x_{d}^{n_{d}} \in \mathfrak{a}(M)$. Since $x_{1}, \ldots, x_{s}$ is a p-standard sequence on $M /\left(x_{s+1}^{n_{s+1}}, \ldots, x_{d}^{n_{d}}\right) M$ of type $s-1$, we obtain $x_{i}^{n_{i}} \in \mathfrak{a}\left(M /\left(x_{i+1}^{n_{i+1}}, \ldots, x_{d}^{n_{d}}\right) M\right)$ for $i \leq s$ by use of the inductive assumption.

The assumptions of Theorem 1.1 assure us of the existence of a p-standard system of parameters.

Theorem 3.5 ([15, Theorem 2.5]). Assume that A satisfies (C1), (C2) and that $M$ satisfies (QU). Then there is a p-standard system of parameters for $M$.

\section{The Highest LOCAL COHOMOLOGY}

Let $A$ be a commutative ring, $M$ an $A$-module and $\boldsymbol{x}=x_{1}, \ldots, x_{d} \in A$. In this section, we consider the highest local cohomology module $E=H_{\boldsymbol{x}}^{d}(M)$.

First we prove the following proposition. It is the dual of a well-known result (see the proof of [7, Lemma 2.4]), but our situation is general. For the sake of completeness, we give a brief proof.

Proposition 4.1. The zeroth Koszul homology $H_{0}(\boldsymbol{x} ; E)$ is zero. If $d>1$, then $H_{1}(\boldsymbol{x} ; E)$ is also zero.

Proof. Since $x_{1}\left[0:_{M} x_{1}\right]=0$ and $x_{1}\left[M / x_{1} M\right]=0$, we obtain an exact sequence

$$
H_{\boldsymbol{x}}^{d-1}\left(M / x_{1} M\right) \rightarrow H_{\boldsymbol{x}}^{d}(M) \stackrel{x_{1}}{\rightarrow} H_{\boldsymbol{x}}^{d}(M) \rightarrow 0 .
$$

That is, $x_{1} E=E$. Therefore $H_{0}(\boldsymbol{x} ; E)=0$. 
If $d>1$, then $H_{x_{2}, \ldots, x_{d}}^{d-1}\left(M / x_{1} M\right)=H_{\boldsymbol{x}}^{d-1}\left(M / x_{1} M\right)$ and we can also show that $x_{2}\left[H_{x_{2}, \ldots, x_{d}}^{d-1}\left(M / x_{1} M\right)\right]=H_{x_{2}, \ldots, x_{d}}^{d-1}\left(M / x_{1} M\right)$. Therefore $x_{2}\left[0:_{E} x_{1}\right]=0:_{E} x_{1}$ and hence $H_{1}\left(x_{1}, x_{2} ; E\right)=0$. The spectral sequence

$$
E_{p q}^{2}=H_{p}\left(x_{3}, \ldots, x_{d} ; H_{q}\left(x_{1}, x_{2} ; E\right)\right) \Rightarrow H_{p+q}(\boldsymbol{x} ; E)
$$

tells us that $H_{1}(\boldsymbol{x} ; E)=0$.

Assume $d>2$. We consider the vanishing of $H_{p}(\boldsymbol{x} ; E)$ for $2 \leq p<d$.

Theorem 4.2. Let $d \geq l \geq 3$. We consider the following three conditions:

(1) $H_{x_{p-1}}^{0} H_{x_{p}, \ldots, x_{d}}^{d-p+1}(M)=0$ for $2 \leq p<l$;

(2) $H_{x_{1}, \ldots, x_{l-2}}^{k} H_{x_{l-1}, \ldots, x_{d}}^{d-l+2}(M)=0$ for $0 \leq k<l-2$;

(3) $H_{p}(\boldsymbol{x} ; E)=0$ for $p<l$.

Then (1) implies (2) and (2) implies (3). If A is a Noetherian ring with Jacobson radical $\mathfrak{m}, M$ a finitely generated $A$-module and $x_{1}, \ldots, x_{d} \in \mathfrak{m}$ a p-standard sequence on $M$, then (3) implies (1).

Proof. $(1) \Rightarrow(2)$ : We show that

$$
H_{x_{p-1}, \ldots, x_{l-2}}^{k} H_{x_{l-1}, \ldots, x_{d}}^{d-l+2}(M)=0 \quad \text { for } k<l-p
$$

by descending induction on $p$. If $p=l-1$, then this claim follows from (1).

Assume that $p<l-1$ and that the claim is true for larger values of $p$. There is an exact sequence

$$
\begin{aligned}
0 \rightarrow H_{x_{p-1}}^{1} H_{x_{p}, \ldots, x_{l-2}}^{k-1} H_{x_{l-1}, \ldots, x_{d}}^{d-l+2}(M) \rightarrow & H_{x_{p-1}, \ldots, x_{l-2}}^{k} H_{x_{l-1}, \ldots, x_{d}}^{d-l+2}(M) \rightarrow \\
& H_{x_{p-1}}^{0} H_{x_{p}, \ldots, x_{l-2}}^{k} H_{x_{l-1}, \ldots, x_{d}}^{d-l+2}(M) \rightarrow 0 .
\end{aligned}
$$

If $k<l-p-1$, then the inductive assumption implies that

$$
H_{x_{p}, \ldots, x_{l-2}}^{k} H_{x_{l-1}, \ldots, x_{d}}^{d-l+2}(M)=0
$$

and

$$
H_{x_{p}, \ldots, x_{l-2}}^{k-1} H_{x_{l-1}, \ldots, x_{d}}^{d-l+2}(M)=0 .
$$

Therefore $H_{x_{p-1}, \ldots, x_{l-2}}^{k} H_{x_{l-1}, \ldots, x_{d}}^{d-l+2}(M)=0$ in view of the exact sequence above. Furthermore the same exact sequence also yields that

$$
\begin{aligned}
H_{x_{p-1}, \ldots, x_{l-2}}^{l-p-1} H_{x_{l-1}, \ldots, x_{d}}^{d-l+2}(M) & =H_{x_{p-1}}^{0} H_{x_{p}, \ldots, x_{l-2}}^{l-p-1} H_{x_{l-1}, \ldots, x_{d}}^{d-l+2}(M) \\
& =H_{x_{p-1}}^{0} H_{x_{p}, \ldots, x_{d}}^{d-p+1}(M)=0 .
\end{aligned}
$$

$(2) \Rightarrow(3)$ : Let $C \bullet$ be the Čech complex of $H_{x_{l-1}, \ldots, x_{d}}^{d-l+2}(M)$ with respect to $x_{1}, \ldots$, $x_{l-2}$, that is, the direct limit of Koszul complexes

$$
K^{\bullet}\left(x_{1}^{m}, \ldots, x_{l-2}^{m} ; H_{x_{l-1}, \ldots, x_{d}}^{d-l+2}(M)\right) .
$$

Then

$$
H^{l-2}\left(C^{\bullet}\right)=H_{x_{1}, \ldots, x_{l-2}}^{l-2} H_{x_{l-1}, \ldots, x_{d}}^{d-l+2}(M)=E
$$

and (2) tells us that

$$
0 \rightarrow C^{0} \rightarrow C^{1} \rightarrow \cdots \rightarrow C^{l-2} \rightarrow E \rightarrow 0
$$


is exact. If $p>0$, then

$$
C^{p}=\bigoplus_{1 \leq i_{1}<\cdots<i_{p} \leq l-2}\left[H_{x_{l-1}, \ldots, x_{d}}^{d-l+2}(M)\right]_{x_{i_{1}} \cdots x_{i_{p}}}
$$

and hence $H_{i}\left(\boldsymbol{x} ; C^{p}\right)=0$ for all $i$. Furthermore, Proposition 4.1 implies that $H_{i}\left(x_{l-1}, \ldots, x_{d} ; C^{0}\right)=0$ for $i=0,1$. The spectral sequence

$$
E_{p q}^{2}=H_{p}\left(x_{1}, \ldots, x_{l-2} ; H_{q}\left(x_{l-1}, \ldots, x_{d} ; C^{0}\right)\right) \Rightarrow H_{p+q}\left(\boldsymbol{x} ; C^{0}\right)
$$

shows that $H_{i}\left(\boldsymbol{x} ; C^{0}\right)=0$ for $i=0,1$. By splitting (4.2.1) into short exact sequences and taking long exact sequences of Koszul cohomologies, we obtain $H_{i}(\boldsymbol{x} ; E)=0$ for $i<l$.

$(3) \Rightarrow(1)$ : Assume that $A$ is Noetherian, $M$ is finitely generated and $x_{1}, \ldots, x_{d}$ is a p-standard sequence on $M$ in the Jacobson radical of $A$. Then

$$
\begin{aligned}
H_{x_{p-1}}^{0} H_{x_{p}, \ldots, x_{d}}^{d-p+1}(M) & =H_{x_{p-1}}^{0}\left(\underset{m}{\operatorname{inj} \lim } M /\left(x_{p}^{m}, \ldots, x_{d}^{m}\right) M\right) \\
& =\underset{m}{\operatorname{inj} \lim } \frac{\left(x_{p}^{m}, \ldots, x_{d}^{m}\right) M: x_{p-1}}{\left(x_{p}^{m}, \ldots, x_{d}^{m}\right) M},
\end{aligned}
$$

because a p-standard sequence is an unconditioned p-sequence. Proposition 2.3 implies that

$$
\left(x_{p}^{m}, \ldots, x_{d}^{m}\right) M: x_{p-1}=\sum_{\Lambda \subset\{p, \ldots, d\}}\left(\prod_{\lambda \in \Lambda} x_{\lambda}^{m-1}\right)\left[\left(x_{\lambda} \mid \lambda \in \Lambda\right) M: x_{p-1}\right] .
$$

As in the proof of Proposition 2.4, we find that

$$
x_{p} \cdots x_{d}\left[\sum_{\Lambda \subsetneq\{p, \ldots, d\}}\left(\prod_{\lambda \in \Lambda} x_{\lambda}^{m-1}\right)\left[\left(x_{\lambda} \mid \lambda \in \Lambda\right) M: x_{p-1}\right]\right] \subset\left(x_{p}^{m+1}, \ldots, x_{d}^{m+1}\right) M .
$$

That is, the image of

$$
\sum_{\Lambda \subsetneq\{p, \ldots, d\}}\left(\prod_{\lambda \in \Lambda} x_{\lambda}^{m-1}\right)\left[\left(x_{\lambda} \mid \lambda \in \Lambda\right) M: x_{p-1}\right]
$$

in

$$
\left(x_{p}^{m+1}, \ldots, x_{d}^{m+1}\right) M: x_{p-1} /\left(x_{p}^{m+1}, \ldots, x_{d}^{m+1}\right) M
$$

is zero. Therefore the natural homomorphism

$$
\frac{\left(x_{p}, \ldots, x_{d}\right) M: x_{p-1}}{\left(x_{p}, \ldots, x_{d}\right) M} \rightarrow \operatorname{inj} \lim \frac{\left(x_{p}^{m}, \ldots, x_{d}^{m}\right) M: x_{p-1}}{\left(x_{p}^{m}, \ldots, x_{d}^{m}\right) M}
$$

is an epimorphism. Thus $H_{x_{p-1}}^{0} H_{x_{p}, \ldots, x_{d}}^{d-p+1}(M)$ is finitely generated.

We show that

$$
H_{x_{p-1}}^{0} H_{x_{p}, \ldots, x_{d}}^{d-p+1}(M)=0
$$

and

$$
H_{k}\left(\boldsymbol{x} ; H_{x_{p}, \ldots, x_{d}}^{d-p+1}(M)\right)=0 \quad \text { for } 0 \leq k<l-p+1
$$

if $2 \leq p<l$. We work by induction on $p$. Assume that $2 \leq p<l$ and

$$
H_{k}\left(\boldsymbol{x} ; H_{x_{p-1}, \ldots, x_{d}}^{d-p+2}(M)\right)=0 \text { for } 0 \leq k<l-p+2 .
$$


There is an exact sequence

$$
\begin{aligned}
& 0 \rightarrow H_{x_{p-1}}^{0} H_{x_{p}, \ldots, x_{d}}^{d-p+1}(M) \rightarrow H_{x_{p}, \ldots, x_{d}}^{d-p+1}(M) \rightarrow \\
& \quad\left[H_{x_{p}, \ldots, x_{d}}^{d-p+1}(M)\right]_{x_{p-1}} \rightarrow H_{x_{p-1}, \ldots, x_{d}}^{d-p+2}(M) \rightarrow 0
\end{aligned}
$$

where we used the isomorphism $H_{x_{p-1}}^{1} H_{x_{p}, \ldots, x_{d}}^{d-p+1}(M) \cong H_{x_{p-1}, \ldots, x_{d}}^{d-p+2}(M)$. Since $d-$ $p+1 \geq 2$, we obtain

$$
H_{k}\left(\boldsymbol{x} ; H_{x_{p}, \ldots, x_{d}}^{d-p+1}(M)\right)=0 \quad \text { for } k=0,1
$$

by using Proposition 4.1 and the spectral sequence

$$
E_{i j}^{2}=H_{i}\left(x_{1}, \ldots, x_{p-1} ; H_{j}\left(x_{p}, \ldots, x_{d} ; H_{x_{p}, \ldots, x_{d}}^{d-p+1}(M)\right)\right) \Rightarrow H_{i+j}\left(\boldsymbol{x} ; H_{x_{p}, \ldots, x_{d}}^{d-p+1}(M)\right) .
$$

On the other hand,

$$
H_{k}\left(\boldsymbol{x} ;\left[H_{x_{p}, \ldots, x_{d}}^{d-p+1}(M)\right]_{x_{p-1}}\right)=0 \text { for all } k .
$$

Therefore $H_{0}\left(\boldsymbol{x} ; H_{x_{p-1}}^{0} H_{x_{p}, \ldots, x_{d}}^{d-p+1}(M)\right)=0$. That is,

$$
H_{x_{p-1}}^{0} H_{x_{p}, \ldots, x_{d}}^{d-p+1}(M)=\boldsymbol{x}\left[H_{x_{p-1}}^{0} H_{x_{p}, \ldots, x_{d}}^{d-p+1}(M)\right] .
$$

We know that $H_{x_{p-1}}^{0} H_{x_{p}, \ldots, x_{d}}^{d-p+1}(M)$ is finitely generated. Therefore it follows from Nakayama's lemma that $H_{x_{p-1}}^{0} H_{x_{p}, \ldots, x_{d}}^{d-p+1}(M)=0$. It then follows from the exact sequence above that

$$
H_{k}\left(\boldsymbol{x} ; H_{x_{p}, \ldots, x_{d}}^{d-p+1}(M)\right)=0 \quad \text { for } 0 \leq k<l-p+1 .
$$

\section{Cousin COMPleXes}

In this section we recall the result of Dibaei and Tousi.

Let $A$ be a Noetherian ring and $M$ a finitely generated $A$-module. For a prime ideal $\mathfrak{p} \in \operatorname{Supp} M$, the $M$-height of $\mathfrak{p}$ is defined to be $\operatorname{ht}_{M} \mathfrak{p}=\operatorname{dim} M_{\mathfrak{p}}$. If $\mathfrak{b} \subset A$ is an ideal such that $M \neq \mathfrak{b} M$, then let ht $\operatorname{lo}_{M} \mathfrak{b}=\inf \left\{\operatorname{ht}_{M} \mathfrak{p} \mid \mathfrak{p} \in \operatorname{Supp} M \cap V(\mathfrak{b})\right\}$.

Definition 5.1. For an integer $l \geq 0$, let $U^{l}(M)=\left\{\mathfrak{p} \in \operatorname{Supp} M \mid \mathrm{ht}_{M} \mathfrak{p} \geq l\right\}$.

The Cousin complex $M^{\bullet}$ of $M$ is defined as follows. Let $M^{-2}=0, M^{-1}=M$ and $d_{M}^{-2}: M^{-2} \rightarrow M^{-1}$ be the zero map. If $p \geq 0$ and $d_{M}^{p-2}: M^{p-2} \rightarrow M^{p-1}$ is given, then we put

$$
M^{p}=\bigoplus_{\mathfrak{p} \in U^{p}(M) \backslash U^{p+1}(M)}\left(\operatorname{coker} d_{M}^{p-2}\right)_{\mathfrak{p}}
$$

If $\xi \in M^{p-1}$ and its image in coker $d_{M}^{p-2}$ is $\bar{\xi}$, then the component of $d_{M}^{p-1}(\xi) \in M^{p}$ in $\left(\operatorname{coker} d_{M}^{p-2}\right)_{\mathfrak{p}}$ is $\bar{\xi} / 1$.

Definition 5.2. A codimension function of $A$ is an integer-valued function $t$ on $\operatorname{Spec} A$ such that ht $\mathfrak{p} / \mathfrak{q}=t(\mathfrak{p})-t(\mathfrak{q})$ whenever $\mathfrak{p} \supset \mathfrak{q}$.

Assume that $A$ has a fundamental dualizing complex $D_{A}^{\bullet}[10]$. Then there is a codimension function $t$ such that $D_{A}^{p}=\bigoplus_{t(\mathfrak{p})=p} E(A / \mathfrak{p})$ where $E(-)$ denotes the injective envelope. Let $t_{0}=\min \{t(\mathfrak{p}) \mid \mathfrak{p} \in \operatorname{Supp} M\}$. Then $\operatorname{Hom}\left(M, D_{A}^{p}\right)=0$ for $p<t_{0}$ and $K_{M}=H^{t_{0}}\left(\operatorname{Hom}\left(M, D_{A}^{\bullet}\right)\right) \neq 0$. We call this the canonical module of $M$. If $A$ is local, then our definition of the canonical module coincides with the usual one. 
The natural quasi-isomorphism $M \rightarrow \operatorname{Hom}\left(\operatorname{Hom}\left(M, D_{A}^{\bullet}\right), D_{A}^{\bullet}\right)$ induces a spectral sequence

$$
E_{2}^{p q}=H^{p}\left(\operatorname{Hom}\left(H^{-q}\left(\operatorname{Hom}\left(M, D_{A}^{\bullet}\right)\right), D_{A}^{\bullet}\right)\right) \Rightarrow E^{p+q}
$$

where $E^{0}=M$ and $E^{n}=0$ whenever $n \neq 0$. Since $\operatorname{Hom}\left(M, D_{A}^{-q}\right)=0$ if $-q<t_{0}$, we find that $E_{2}^{p q}=0$ if $q>-t_{0}$. Since $H^{-q}\left(\operatorname{Hom}\left(M, D_{A}^{\bullet}\right)\right)$ is a subquotient of $\operatorname{Hom}\left(M, \bigoplus_{t(\mathfrak{p})=-q} E(A / \mathfrak{p})\right)$, the support of $H^{-q}\left(\operatorname{Hom}\left(M, D_{A}^{\bullet}\right)\right)$ is contained in $\{\mathfrak{p} \in \operatorname{Supp} M \mid t(\mathfrak{p}) \geq-q\}$. Therefore $\operatorname{Hom}\left(H^{-q}\left(\operatorname{Hom}\left(M, D_{A}^{\bullet}\right)\right), D_{A}^{p}\right)=0$ if $p<-q$ and hence $E_{2}^{p q}=0$ if $p+q<0$.

The spectral sequence gives a filtration

$$
M=F^{t_{0}} \supset F^{t_{0}+1} \supset \cdots
$$

of $E^{0}=M$ such that $F^{n}=0$ for sufficiently large $n$ and $F^{p} / F^{p+1} \cong E_{\infty}^{p,-p}$ for all $p$. Let $h_{M}: M \rightarrow E_{2}^{t_{0},-t_{0}}$ be the composition of the natural epimorphism $M=F^{t_{0}} \rightarrow E_{\infty}^{t_{0},-t_{0}}$ and the inclusion $E_{\infty}^{t_{0},-t_{0}} \hookrightarrow E_{2}^{t_{0},-t_{0}}$. The following lemma was given by Aoyama. (See the proof of Theorem 4.2 of [1]). For the sake of completeness, we give a brief proof.

Lemma 5.3. With the same notation as above, we have

(1) $t(\mathfrak{p}) \geq t_{0}+1$ for any $\mathfrak{p} \in \operatorname{Supp}\left(\operatorname{ker} h_{M}\right)$;

(2) $t(\mathfrak{p}) \geq t_{0}+2$ for any $\mathfrak{p} \in \operatorname{Supp}\left(\operatorname{coker} h_{M}\right)$.

Proof. (1): Let $p>t_{0}$. If $\mathfrak{p} \in \operatorname{Supp} E_{\infty}^{p,-p}$, then $t(\mathfrak{p}) \geq p>t_{0}$ because $E_{\infty}^{p,-p}$ is a subquotient of $\operatorname{Hom}\left(H^{p}\left(\operatorname{Hom}\left(M, D_{A}^{\bullet}\right)\right), D_{A}^{p}\right)$. Since $F^{n}=0$ for sufficiently large $n$ and $E_{\infty}^{p,-p} \cong F^{p} / F^{p+1}$, we can prove that $t(\mathfrak{p}) \geq p$ if $\mathfrak{p} \in \operatorname{Supp} F^{p}$ by descending induction on $p$. In particular, $t(\mathfrak{p}) \geq t_{0}+1$ if $\mathfrak{p} \in \operatorname{Supp}\left(\operatorname{ker} h_{M}\right)=\operatorname{Supp} F^{t_{0}+1}$.

(2): If $r \geq 2$, then

$$
0 \rightarrow E_{r+1}^{t_{0},-t_{0}} \rightarrow E_{r}^{t_{0},-t_{0}} \rightarrow E_{r}^{t_{0}+r,-t_{0}-r+1}
$$

is exact and hence

$$
0 \rightarrow E_{r+1}^{t_{0},-t_{0}} / E_{\infty}^{t_{0},-t_{0}} \rightarrow E_{r}^{t_{0},-t_{0}} / E_{\infty}^{t_{0},-t_{0}} \rightarrow E_{r}^{t_{0}+r,-t_{0}-r+1}
$$

is also. If $\mathfrak{p} \in \operatorname{Supp} E_{r}^{t_{0}+r,-t_{0}-r+1}$, then $t(\mathfrak{p}) \geq t_{0}+r$ because $E_{r}^{t_{0}+r,-t_{0}-r+1}$ is a subquotient of $\operatorname{Hom}\left(H^{t_{0}+r-1}\left(\operatorname{Hom}\left(M, D_{A}^{\bullet}\right)\right), D_{A}^{t_{0}+r}\right)$. By descending induction on $r$, we obtain that $t(\mathfrak{p}) \geq t_{0}+r$ if $\mathfrak{p} \in \operatorname{Supp} E_{r}^{t_{0},-t_{0}} / E_{\infty}^{t_{0},-t_{0}}$. In particular, $t(\mathfrak{p}) \geq t_{0}+2$ if $\mathfrak{p} \in \operatorname{Supp} E_{2}^{t_{0},-t_{0}} / E_{\infty}^{t_{0},-t_{0}}=\operatorname{Supp}\left(\operatorname{coker} h_{M}\right)$.

The following theorem is a slight refinement of Theorem 3.2 of [5].

Theorem 5.4. Assume that $A$ has a dualizing complex $D_{A}^{\bullet}$ with codimension function $t$ and that $t$ has the constant value $t_{0}$ on all the minimal primes of $A$. Then the Cousin complex $M^{\bullet}$ of $M$ coincides with

$$
0 \rightarrow M \stackrel{\iota h_{M}}{\longrightarrow} \operatorname{Hom}\left(K_{M}, D_{A}^{t_{0}}\right) \rightarrow \operatorname{Hom}\left(K_{M}, D_{A}^{t_{0}+1}\right) \rightarrow \cdots
$$

where $\iota: H^{t_{0}}\left(\operatorname{Hom}\left(K_{M}, D_{A}^{\bullet}\right)\right) \rightarrow \operatorname{Hom}\left(K_{M}, D_{A}^{t_{0}}\right)$ is the inclusion. In particular, all the cohomology modules of $M^{\bullet}$ are finitely generated.

Proof. It follows from the assumptions that $\mathrm{ht}_{M} \mathfrak{p}=t(\mathfrak{p})-t_{0}$ for all $\mathfrak{p} \in \operatorname{Supp} M$ and $U^{l}(M)=\left\{\mathfrak{p} \in \operatorname{Supp} M \mid t(\mathfrak{p}) \geq t_{0}+l\right\}$ for all $l$.

We apply Proposition 2.1 of [4] to the complex (5.4.1). Let $X^{-1}=M, X^{l}=$ $\operatorname{Hom}\left(K_{M}, D_{A}^{t_{0}+l}\right), e^{-1}=\iota h_{M}$ and $e^{l}: X^{l} \rightarrow X^{l+1}$ be the natural homomorphism induced by differentials of $D_{A}^{\bullet}$ for all $l \geq 0$. 
Since $D_{A}^{t_{0}+l}=\bigoplus_{t(\mathfrak{p})=t_{0}+l} E(A / \mathfrak{p})$, we obtain that

(a) $\operatorname{Supp} X^{l} \subset U^{l}(M)$;

(b) the natural homomorphism $X^{l} \rightarrow \bigoplus_{\mathfrak{p} \in U^{l}(M) \backslash U^{l+1}(M)}\left(X^{l}\right)_{\mathfrak{p}}$ is an isomorphism

for all $l \geq 0$. See the proof of Theorem 1.4 of [5].

It follows from Lemma 5.3 that

$$
\operatorname{Supp} H^{-1}\left(X^{\bullet}\right)=\operatorname{Supp}\left(\operatorname{ker} h_{M}\right) \subset U^{1}(M)
$$

and

$$
\operatorname{Supp} H^{0}\left(X^{\bullet}\right)=\operatorname{Supp}\left(\operatorname{coker} h_{M}\right) \subset U^{2}(M) .
$$

Since the canonical module $K_{M}$ satisfies $\left(S_{2}\right)$, we have

$$
\operatorname{Supp}\left(\operatorname{coker} e^{l-1}\right) \subset U^{l+1}(M)
$$

and

$$
\operatorname{Supp} H^{l}\left(X^{\bullet}\right) \subset U^{l+2}(M)
$$

for all $l>0$. See the proof of Theorem 1.4 of [5]. If $\mathfrak{p} \in U^{0}(M) \backslash U^{1}(M)$, then

$$
\left(e^{-1}\right)_{\mathfrak{p}}: M_{\mathfrak{p}} \rightarrow \operatorname{Hom}\left(K_{M}, D_{A}^{t_{0}}\right)_{\mathfrak{p}}=\operatorname{Hom}\left(\operatorname{Hom}\left(M_{\mathfrak{p}}, E(A / \mathfrak{p})\right), E(A / \mathfrak{p})\right)
$$

is an isomorphism because $\operatorname{dim} M_{\mathfrak{p}}=0$. Therefore $\operatorname{Supp}\left(\right.$ coker $\left.e^{-1}\right) \subset U^{1}(M)$.

Thus $X^{\bullet}$ satisfies the assumptions of Proposition 2.1 of [4].

Theorem 5.5. Let $A$ be a Noetherian local ring satisfying $(\mathrm{C} 1),(\mathrm{C} 2)$ and $M a$ finitely generated A-module satisfying (QU). Then all the cohomology modules of the Cousin complex $M^{\bullet}$ of $M$ are finitely generated.

Proof. The completion $\hat{A}$ has a dualizing complex with codimension function $t(\mathfrak{p})=$ $-\operatorname{dim} A / \mathfrak{p}$.

Furthermore, since $A$ is universally catenary, $A / \mathfrak{p}$ is quasi-unmixed for any $\mathfrak{p} \in$ Spec $A$ [18]. Therefore the completion $\hat{M}$ also satisfies (QU).

By applying Theorem 5.4 to a finitely generated $\hat{A}$-module $\hat{M}$, we find that $H^{p}\left(\hat{M}^{\bullet}\right)$ is a finitely generated $\hat{A}$-module for each $p$. It follows from Theorem 3.5 of [17] that $H^{p}\left(M^{\bullet}\right) \otimes \hat{A} \cong H^{p}\left(\hat{M}^{\bullet}\right)$ for each $p$. Therefore $H^{p}\left(M^{\bullet}\right)$ is finitely generated.

\section{The PRoof of TheOREM 1.1}

We start the proof of Theorem 1.1. Let $A$ be a Noetherian ring, $M$ a finitely generated $A$-module and $M^{\bullet}$ the Cousin complex of $M$.

We need the following lemma to prove Theorem 1.1. We also use this to prove Theorem 1.2.

Lemma 6.1. Let $\boldsymbol{x}=x_{1}, \ldots, x_{n}$ be a sequence in A such that $\mathrm{ht}_{M}\left(x_{1}, \ldots, x_{n}\right)=n$ and $\mathfrak{a}^{\prime}=\prod_{-1 \leq q<n-1}$ ann $H^{q}\left(M^{\bullet}\right)$. Then

$$
\left(x_{1}, \ldots, x_{n-1}\right) M: x_{n} \subset\left(x_{1}, \ldots, x_{n-1}\right) M: \mathfrak{a}^{\prime} .
$$

If $x_{1}, \ldots, x_{n}$ are in the Jacobson radical of $A$, then

$$
\left(x_{1}, \ldots, x_{p-1}\right) M: x_{p} \subset\left(x_{1}, \ldots, x_{p-1}\right) M: \mathfrak{a}^{\prime}
$$

for any $1 \leq p \leq n$.

The proof is quite similar to that of Lemma 3.2. 
Proof. Let $K^{\bullet}=K^{\bullet}(\boldsymbol{x} ; A)$ be the Koszul complex. The double complex $M^{\bullet} \otimes K^{\bullet}$ gives two spectral sequences

$$
\begin{gathered}
{ }^{\prime} E_{2}^{p q}=H^{p}\left(H^{q}\left(\boldsymbol{x} ; M^{\bullet}\right)\right) \Rightarrow H^{p+q}\left(M^{\bullet} \otimes K^{\bullet}\right), \\
{ }^{\prime \prime} E_{2}^{p q}=H^{p}\left(\boldsymbol{x} ; H^{q}\left(M^{\bullet}\right)\right) \Rightarrow H^{p+q}\left(M^{\bullet} \otimes K^{\bullet}\right) .
\end{gathered}
$$

Since ${ }^{\prime} E_{2}^{p q}$ is the cohomology of

$$
\cdots \rightarrow H^{q}\left(\boldsymbol{x} ; M^{p-1}\right) \rightarrow H^{q}\left(\boldsymbol{x} ; M^{p}\right) \rightarrow H^{q}\left(\boldsymbol{x} ; M^{p+1}\right) \rightarrow \cdots,
$$

we find that ' $E_{2}^{p q}=0$ if $p<-1$ or if $q<0$. Let $\mathfrak{p} \subset A$ be a prime ideal such that $p=\mathrm{ht}_{M} \mathfrak{p}<n$. Then $\left(x_{1}, \ldots, x_{n}\right) \not \subset \mathfrak{p}$ and hence $\left(\text { coker } d_{M}^{p-2}\right)_{\mathfrak{p}} \otimes K^{\bullet}$ is exact. Therefore, if $0 \leq p<n$, then $H^{q}\left(\boldsymbol{x} ; M^{p}\right)=0$ and hence ${ }^{\prime} E_{2}^{p q}=0$. Furthermore ${ }^{\prime} E_{2}^{-1, q}=H^{q}\left(\boldsymbol{x} ; M^{-1}\right)=H^{q}(\boldsymbol{x} ; M)$. Since ${ }^{\prime} E_{2}^{p, n-p-2}={ }^{\prime} E_{2}^{p, n-p-1}=0$ whenever $p \neq-1$, we find that $H^{n-2}\left(M^{\bullet} \otimes K^{\bullet}\right)={ }^{\prime} E_{\infty}^{-1, n-1}=\cdots={ }^{\prime} E_{3}^{-1, n-1}={ }^{\prime} E_{2}^{-1, n-1}=$ $H^{n-1}(\boldsymbol{x} ; M)$. On the other hand, " $E_{2}^{p q}=0$ if $p<0$ or if $q<-1$. Furthermore ${ }^{\prime \prime} E_{2}^{p q}$ is annihilated by ann $H^{q}\left(M^{\bullet}\right)$ and there is a filtration

$$
H^{n-2}\left(M^{\bullet} \otimes K^{\bullet}\right)=F^{0} \supset F^{1} \supset \cdots \supset F^{n-1} \supset F^{n}=0
$$

of $H^{n-2}\left(M^{\bullet} \otimes K^{\bullet}\right)$ such that $F^{p} / F^{p+1} \cong{ }^{\prime \prime} E_{\infty}^{p, n-p-2}$. Therefore $H^{n-2}\left(M^{\bullet} \otimes K^{\bullet}\right)$ is annihilated by $\mathfrak{a}^{\prime}$. Since

$$
H^{n-1}(\boldsymbol{x} ; M) \rightarrow M /\left(x_{1}, \ldots, x_{n-1}\right) M \stackrel{x_{n}}{\longrightarrow} M /\left(x_{1}, \ldots, x_{n-1}\right) M
$$

is exact, we obtain that $\mathfrak{a}^{\prime}\left[\left(x_{1}, \ldots, x_{n-1}\right) M: x_{n} /\left(x_{1}, \ldots, x_{n-1}\right) M\right]=0$.

If $x_{1}, \ldots, x_{n}$ are in the Jacobson radical, then it follows from Krull's intersection theorem that

$$
\begin{aligned}
\left(x_{1}, \ldots, x_{p-1}\right) M: x_{p} & \subset \bigcap_{m}\left[\left(x_{1}, \ldots, x_{p-1}, x_{p+1}^{m}, \ldots, x_{n}^{m}\right) M: x_{p}\right] \\
& \subset \bigcap_{m}\left[\left(x_{1}, \ldots, x_{p-1}, x_{p+1}^{m}, \ldots, x_{n}^{m}\right) M: \mathfrak{a}^{\prime}\right] \\
& \left.\subset \bigcap_{m}\left(x_{1}, \ldots, x_{p-1}, x_{p+1}^{m}, \ldots, x_{n}^{m}\right) M\right]: \mathfrak{a}^{\prime} \\
& =\left(x_{1}, \ldots, x_{p-1}\right) M: \mathfrak{a}^{\prime} .
\end{aligned}
$$

From now on, we assume that $A$ satisfies (C1)-(C3) and that $M$ satisfies (QU). Let $Z^{l}(M)=\bigcup_{p \geq l} \operatorname{Supp} H^{p}\left(M^{\bullet}\right)$. It is known that $Z^{l}(M) \subset U^{l+2}(M)$ for all $l[22$, (2.7)]. We need the following lemma to study $Z^{l}(M)$.

Lemma 6.2. Let $\mathfrak{p} \in \operatorname{Supp} M$ be a prime ideal of $M$-height $d>0, \boldsymbol{x}=x_{1}, \ldots, x_{d}$ a system of parameters for $M_{\mathfrak{p}}$ and $l>0$ an integer. Then the following statements are equivalent:

(1) $H^{q}\left(M^{\bullet}\right)_{\mathfrak{p}}=0$ for all $q>l$;

(2) $H^{q}\left(\boldsymbol{x} ; H_{\mathfrak{p} A_{\mathfrak{p}}}^{d}\left(M_{\mathfrak{p}}\right)\right)=0$ for all $q>l$.

Proof. Since the construction of Cousin complexes commutes with localization, we may assume that $A$ is a local ring and $\mathfrak{p}$ the maximal ideal of $A$. It is known that $M^{d} \cong H_{\mathfrak{p}}^{d}(M)[24]$. 
Let $K^{\bullet}=K^{\bullet}(\boldsymbol{x} ; A)$ be the Koszul complex. The double complex $M^{\bullet} \otimes K^{\bullet}$ gives two spectral sequences

$$
\begin{gathered}
{ }^{\prime} E_{2}^{p q}=H^{p}\left(H^{q}\left(\boldsymbol{x} ; M^{\bullet}\right)\right) \Rightarrow H^{p+q}\left(M^{\bullet} \otimes K^{\bullet}\right), \\
{ }^{\prime \prime} E_{2}^{p q}=H^{p}\left(\boldsymbol{x} ; H^{q}\left(M^{\bullet}\right)\right) \Rightarrow H^{p+q}\left(M^{\bullet} \otimes K^{\bullet}\right) .
\end{gathered}
$$

Since $M^{p}=\bigoplus_{\mathrm{ht}_{M} \mathfrak{q}=p}\left(\operatorname{coker} d_{M}^{p-2}\right)_{\mathfrak{q}}$, we obtain that ${ }^{\prime} E_{2}^{p q}=0$ whenever $p \neq-1, d$. Therefore $H^{d+q}\left(M^{\bullet} \otimes K^{\bullet}\right)=H^{q}\left(\boldsymbol{x} ; M^{d}\right)=H^{q}\left(\boldsymbol{x} ; H_{\mathfrak{p}}^{d}(M)\right)$ for $q>0$.

If $H^{q}\left(M^{\bullet}\right)=0$ for $q>l$, then " $E_{2}^{p q}=0$ for $q>l$ and hence $H^{d+q}\left(K^{\bullet} \otimes M^{\bullet}\right)=0$ for $q>l$. Thus (1) implies (2).

Assume that (2) holds. If there is $l^{\prime}>l$ such that $H^{l^{\prime}}\left(M^{\bullet}\right) \neq 0$ and $H^{q}\left(M^{\bullet}\right)=$ 0 for $q>l^{\prime}$, then ${ }^{\prime \prime} E_{2}^{d, l^{\prime}}=H^{l^{\prime}}\left(M^{\bullet}\right) / \boldsymbol{x} H^{l^{\prime}}\left(M^{\bullet}\right) \neq 0$. Indeed, it follows from Theorem 5.5 that $H^{l^{\prime}}\left(M^{\bullet}\right)$ is finitely generated. Thus

$$
H^{l^{\prime}}\left(\boldsymbol{x} ; H_{\mathfrak{p}}^{d}(M)\right)=H^{d+l^{\prime}}\left(M^{\bullet} \otimes K^{\bullet}\right)={ }^{\prime \prime} E_{2}^{d, l^{\prime}} \neq 0,
$$

which is a contradiction.

We prove the following proposition by induction on $l$.

Proposition 6.3. If $l \geq-1$, then

$\left(A_{l}\right)$ for any finitely generated $A$-module $M$ satisfying $(\mathrm{QU}), \overline{Z^{l}(M)} \subset U^{l+2}(M)$;

$\left(B_{l}\right)$ for any finitely generated $A$-module $M$ satisfying (QU), $H^{p}\left(M^{\bullet}\right)$ is finitely generated whenever $p \leq l$.

Indeed, $Z^{-1}(M)$ is equal to the non-Cohen-Macaulay locus of $M[22$, Theorem 4.7]. Since $A$ satisfies (C3), $Z^{-1}(M)$ is a closed subset of $\operatorname{Spec} A$ [7, Proposition 6.11.8]. Therefore $\overline{Z^{-1}(M)}=Z^{-1}(M) \subset U^{1}(M)$. Thus $\left(A_{-1}\right)$ holds.

Naturally $H^{-1}\left(M^{\bullet}\right) \subset M$ is finitely generated. Thus $\left(B_{-1}\right)$ holds.

Let $N=M / H^{-1}\left(M^{\bullet}\right)$. Then $N$ also satisfies (QU). Because of the construction of the Cousin complex, $H^{-1}\left(N^{\bullet}\right)=0$ and $H^{p}\left(N^{\bullet}\right)=H^{p}\left(M^{\bullet}\right)$ for $p \geq 0$. Therefore $Z^{0}(M)=Z^{-1}(N)$ is closed and $\overline{Z^{0}(M)}=Z^{0}(M) \subset U^{2}(M)$. Thus $\left(A_{0}\right)$ holds.

Claim 1. If $l \geq 0$, then $\left(A_{l}\right)$ and $\left(B_{l-1}\right)$ imply $\left(B_{l}\right)$.

Proof. Let $\mathfrak{b}$ be a defining ideal of $\overline{Z^{l}(M)}$; that is, $V(\mathfrak{b})=\overline{Z^{l}(M)}$. Let $F_{\bullet}$ be a free resolution of $A / \mathfrak{b}$. The double complex $\operatorname{Hom}\left(F_{\bullet}, M^{\bullet}\right)$ gives two spectral sequences

$$
\begin{aligned}
{ }^{\prime} E_{2}^{p q} & =H^{p}\left(\operatorname{Ext}_{A}^{q}\left(A / \mathfrak{b}, M^{\bullet}\right)\right) \Rightarrow H^{p+q}\left(\operatorname{Hom}\left(F_{\bullet}, M^{\bullet}\right)\right), \\
{ }^{\prime \prime} E_{2}^{p q} & =\operatorname{Ext}_{A}^{p}\left(A / \mathfrak{b}, H^{q}\left(M^{\bullet}\right)\right) \Rightarrow H^{p+q}\left(\operatorname{Hom}\left(F_{\bullet}, M^{\bullet}\right)\right) .
\end{aligned}
$$

Since $\left(A_{l}\right)$ holds, $\mathrm{ht}_{M} \mathfrak{b} \geq l+2$. Since $M^{p}=\bigoplus_{\mathrm{ht}_{M} \mathfrak{p}=p}\left(\operatorname{coker} d_{M}^{p-2}\right)_{\mathfrak{p}}$, we find that ${ }^{\prime} E_{2}^{p q}=0$ if $0 \leq p \leq l+1$. Therefore $H^{l}\left(\operatorname{Hom}\left(F_{\bullet}, M^{\bullet}\right)\right)={ }^{\prime} E_{2}^{-1, l+1}=$ $\operatorname{Ext}_{A}^{l+1}(A / \mathfrak{b}, M)$ is finitely generated and hence $" E_{\infty}^{0 l}$ is also. On the other hand, it follows from $\left(B_{l-1}\right)$ that ${ }^{\prime \prime} E_{2}^{p q}$ is finitely generated if $q<l$. By descending induction on $r$, we find that " $E_{r}^{0 l}$ is finitely generated for $r \geq 2$. In particular, ${ }^{\prime \prime} E_{2}^{0 l}=\operatorname{Hom}\left(A / \mathfrak{b}, H^{l}\left(M^{\bullet}\right)\right)$ is finitely generated.

Since $\operatorname{Supp} H^{l}\left(M^{\bullet}\right) \subset V(\mathfrak{b}), \operatorname{Ass} H^{l}\left(M^{\bullet}\right)=\operatorname{Ass} \operatorname{Hom}\left(A / \mathfrak{b}, H^{l}\left(M^{\bullet}\right)\right)$ is a finite set. Since $H^{l}\left(M^{\bullet}\right)$ is locally finitely generated, there is an integer $m$ such that $\mathfrak{b}^{m} H^{l}\left(M^{\bullet}\right)_{\mathfrak{p}}=0$ for any associated prime $\mathfrak{p}$ of $H^{l}\left(M^{\bullet}\right)$. Then $\mathfrak{b}^{m} H^{l}\left(M^{\bullet}\right)=0$. 
Since $\mathfrak{b}^{m}$ is also a defining ideal of $\overline{Z^{l}(M)}$, we obtain that

$$
H^{l}\left(M^{\bullet}\right) \cong \operatorname{Hom}\left(A / \mathfrak{b}^{m}, H^{l}\left(M^{\bullet}\right)\right)
$$

is finitely generated.

We already know that $\left(A_{0}\right)$ and $\left(B_{-1}\right)$ hold. Therefore $\left(B_{0}\right)$ holds.

Let $L=\operatorname{ker} d_{M}^{0}$. Then it follows from $\left(B_{0}\right)$ that $L$ is finitely generated. Since $L \subset M^{0}, L$ also satisfies (QU). Furthermore $H^{-1}\left(L^{\bullet}\right)=H^{0}\left(L^{\bullet}\right)=0$ and $H^{p}\left(L^{\bullet}\right)=$ $H^{p}\left(M^{\bullet}\right)$ for $p>1$. Therefore $Z^{1}(M)=Z^{-1}(L)$ is closed. Thus both $\left(A_{1}\right)$ and $\left(B_{1}\right)$ hold.

The proof of Proposition 6.3 will be completed if we prove the following.

Claim 2. If $l>0$, then $\left(A_{l}\right)$ and $\left(B_{l}\right)$ imply $\left(A_{l+1}\right)$.

Proof. Since $H^{p}\left(M^{\bullet}\right)$ is finitely generated and $\operatorname{Supp} H^{p}\left(M^{\bullet}\right) \subset U^{p+2}(M)$, we find that $\mathrm{ht}_{M}$ ann $H^{p}\left(M^{\bullet}\right) \geq p+2$ for $-1 \leq p \leq l$. There is $y \in \prod_{p=-1}^{l}$ ann $H^{p}\left(M^{\bullet}\right)$ such that $\operatorname{ht}_{M} y A=1$ and $Z^{-1}(M) \subset V(y A)$. Then $M / y^{2} M$ also satisfies (QU) and $U^{l+2}\left(M / y^{2} M\right) \subset U^{l+3}(M)$. We want to show that $Z^{l+1}(M) \subset Z^{l}\left(M / y^{2} M\right)$.

Let $\mathfrak{p} \in \operatorname{Supp} M \backslash Z^{l}\left(M / y^{2} M\right)$ and $d=\mathrm{ht}_{M} \mathfrak{p}$. It is enough to show that $\mathfrak{p} \notin Z^{l+1}(M)$. If $y \notin \mathfrak{p}$, then $M_{\mathfrak{p}}$ is Cohen-Macaulay and hence $\mathfrak{p} \notin Z^{l+1}(M)$.

Assume that $y \in \mathfrak{p}$. Passing to the localization, we may assume that $A$ is a local ring and $\mathfrak{p}$ the maximal ideal of $A$. Since $A$ is local, $M^{d}=$ coker $d_{M}^{d-2}$. Therefore $H^{d-1}\left(M^{\bullet}\right)=H^{d}\left(M^{\bullet}\right)=0$. That is, $\mathfrak{p} \notin Z^{l+1}(M)$ if $d \leq l+2$.

Assume that $d>l+2$. Then there is a p-standard system of parameters $\boldsymbol{x}=x_{1}, \ldots, x_{d-1}$ of type $d-2$ for $M / y^{2} M$. Since $\mathfrak{p} \notin Z^{l}\left(M / y^{2} M\right)$, it follows from Lemma 6.2 that $H^{p}\left(\boldsymbol{x} ; H_{\mathfrak{p}}^{d-1}\left(M / y^{2} M\right)\right)=0$ for $p>l-1$. It follows from Theorem 4.2 that

$$
H_{x_{1}, \ldots, x_{d-l-2}}^{k} H_{x_{d-l-1}, \ldots, x_{d-1}}^{l+1}\left(M / y^{2} M\right)=0 \quad \text { for } k<d-l-2 .
$$

By Lemma 6.1, we have

$$
\left(x_{d-l-1}^{m}, \ldots, x_{d-1}^{m}\right) M: y^{n+1}=\left(x_{d-l-1}^{m}, \ldots, x_{d-1}^{m}\right) M: y \quad \text { for any } m, n>0 .
$$

Therefore $y$ is a non-zero divisor on

$$
L=\operatorname{inj} \lim _{m} M /\left(x_{d-l-1}^{m}, \ldots, x_{d-1}^{m}\right) M: y .
$$

Furthermore, by (6.3.1), we obtain

$$
\left(x_{d-l-1}^{m}, \ldots, x_{d-1}^{m}, y^{n+1}\right) M: y^{n}=\left(x_{d-l-1}^{m}, \ldots, x_{d-1}^{m}\right) M: y+y M
$$

and

$$
\begin{aligned}
& \left(x_{d-l-1}^{m}, \ldots, x_{d-1}^{m}\right) M: y \cap\left(x_{d-l-1}^{m}, \ldots, x_{d-1}^{m}, y^{n}\right) M \\
& \quad=\left(x_{d-l-1}^{m}, \ldots, x_{d-1}^{m}\right) M+y^{n}\left[\left(x_{d-l-1}^{m}, \ldots, x_{d-1}^{m}\right) M: y^{n+1}\right] \\
& \quad=\left(x_{d-l-1}^{m}, \ldots, x_{d-1}^{m}\right) M .
\end{aligned}
$$

Therefore, we obtain two exact sequences:

$$
\begin{aligned}
& 0 \rightarrow M /\left[\left(x_{d-l-1}^{m}, \ldots, x_{d-1}^{m}\right) M: y+y M\right] \rightarrow \\
& \quad M /\left(x_{d-l-1}^{m}, \ldots, x_{d-1}^{m}, y^{n+1}\right) M \rightarrow M /\left(x_{d-l-1}^{m}, \ldots, x_{d-1}^{m}, y^{n}\right) M \rightarrow 0
\end{aligned}
$$


and

$$
\begin{aligned}
0 \rightarrow & \left(x_{d-l-1}^{m}, \ldots, x_{d-1}^{m}\right) M: y /\left(x_{d-l-1}^{m}, \ldots, x_{d-1}^{m}\right) M \rightarrow \\
& \quad M /\left(x_{d-l-1}^{m}, \ldots, x_{d-1}^{m}, y^{n}\right) M \rightarrow M /\left[\left(x_{d-l-1}^{m}, \ldots, x_{d-1}^{m}\right) M: y+y^{n} M\right] \rightarrow 0
\end{aligned}
$$

for any $m, n>0$. By taking the direct limit, we have the exact sequences

$$
0 \rightarrow L / y L \rightarrow H_{x_{d-l-1}, \ldots, x_{d-1}}^{l+1}\left(M / y^{n+1} M\right) \rightarrow H_{x_{d-l-1}, \ldots, x_{d-1}}^{l+1}\left(M / y^{n} M\right) \rightarrow 0
$$

and

$$
0 \rightarrow Q \rightarrow H_{x_{d-l-1}, \ldots, x_{d-1}}^{l+1}\left(M / y^{n} M\right) \rightarrow L / y^{n} L \rightarrow 0
$$

for any $n>0$, where

$$
Q=\operatorname{inj} \lim _{m}\left(x_{d-l-1}^{m}, \ldots, x_{d-1}^{m}\right) M: y /\left(x_{d-l-1}^{m}, \ldots, x_{d-1}^{m}\right) M .
$$

If

$$
H_{x_{1}, \ldots, x_{d-l-2}}^{t} H_{x_{d-l-1}, \ldots, x_{d-1}}^{l+1}(M / y M) \neq 0 \quad \text { for some } t<d-l-2
$$

and

$$
H_{x_{1}, \ldots, x_{d-l-2}}^{k} H_{x_{d-l-1}, \ldots, x_{d-1}}^{l+1}(M / y M)=0 \text { for } k<t,
$$

then $H_{x_{1}, \ldots, x_{d-l-2}}^{t+1}(L / y L) \neq 0$ and $H_{x_{1}, \ldots, x_{d-l-2}}^{k}(L / y L)=0$ for $k<t+1$, because (6.3.2) induces an exact sequence

$$
\begin{aligned}
H_{x_{1}, \ldots, x_{d-l-2}}^{k-1} H_{x_{d-l-1}, \ldots, x_{d-1}}^{l+1}(M / y M) & \rightarrow \\
H_{x_{1}, \ldots, x_{d-l-2}}^{k}(L / y L) & \rightarrow H_{x_{1}, \ldots, x_{d-l-2}}^{k} H_{x_{d-l-1}, \ldots, x_{d-1}}^{l+1}\left(M / y^{2} M\right) .
\end{aligned}
$$

Therefore $H_{x_{1}, \ldots, x_{d-l-2}}^{t}(Q) \neq 0$ and $H_{x_{1}, \ldots, x_{d-l-2}}^{k}(Q)=0$ for $k<t$, because (6.3.3) induces an exact sequence

$$
H_{x_{1}, \ldots, x_{d-l-2}}^{k-1}(L / y L) \rightarrow H_{x_{1}, \ldots, x_{d-l-2}}^{k}(Q) \rightarrow H_{x_{1}, \ldots, x_{d-l-2}}^{k} H_{x_{d-l-1}, \ldots, x_{d-1}}^{l+1}(M / y M) .
$$

Since $0 \rightarrow L / y L \rightarrow L / y^{2} L \rightarrow L / y L \rightarrow 0$ is exact, $H_{x_{1}, \ldots, x_{d-l-2}}^{t+1}\left(L / y^{2} L\right) \neq 0$ and $H_{x_{1}, \ldots, x_{d-l-2}}^{k}\left(L / y^{2} L\right)=0$ for $k<t+1$. Since

$$
H_{x_{1}, \ldots, x_{d-l-2}}^{t-1}\left(L / y^{2} L\right) \rightarrow H_{x_{1}, \ldots, x_{d-l-2}}^{t}(Q) \rightarrow H_{x_{1}, \ldots, x_{d-l-2}}^{t} H_{x_{d-l-1}, \ldots, x_{d-1}}^{l+1}\left(M / y^{2} M\right)
$$

is exact, $H_{x_{1}, \ldots, x_{d-l-2}}^{t}(Q)=0$, which is a contradiction.

Thus

$$
H_{x_{1}, \ldots, x_{d-l-2}}^{k} H_{x_{d-l-1}, \ldots, x_{d-1}}^{l+1}(M / y M)=0 \quad \text { for } k<d-l-2
$$

and

$$
H_{x_{1}, \ldots, x_{d-l-2}}^{k}(L / y L)=0 \quad \text { for } k<d-l-2 .
$$

By induction on $n$, we obtain

$$
H_{x_{1}, \ldots, x_{d-l-2}}^{k} H_{x_{d-l-1}, \ldots, x_{d-1}}^{l+1}\left(M / y^{n} M\right)=0 \quad \text { for } k<d-l-2
$$

whenever $n \geq 2$.

By taking the direct limit, we have

$$
H_{x_{1}, \ldots, x_{d-l-2}}^{k} H_{x_{d-l-1}, \ldots, x_{d-1}, y}^{l+2}(M)=H_{x_{1}, \ldots, x_{d-l-2}}^{k} H_{x_{d-l-1}, \ldots, x_{d-1}}^{l+1} H_{y}^{1}(M)=0
$$

for $k<d-l-2$. It follows from Theorem 4.2 that

$$
H_{p}\left(x_{1}, \ldots, x_{d-1}, y ; H_{\mathfrak{p}}^{d}(M)\right)=0 \text { for } p<d-l \text {. }
$$


Therefore $\mathfrak{p} \notin Z^{l+1}(M)$ by Lemma 6.2 . Thus we obtain that

$$
Z^{l+1}(M) \subset Z^{l}\left(M / y^{2} M\right) .
$$

It follows from $\left(A_{l}\right)$, applied to $M / y^{2} M$ and the above, that

$$
\overline{Z^{l+1}(M)} \subset \overline{Z^{l}\left(M / y^{2} M\right)} \subset U^{l+2}\left(M / y^{2} M\right) \subset U^{l+3}(M) .
$$

The proof of Claim 2 is completed.

We have almost finished the proof of Theorem 1.1. Indeed, by $\left(A_{l}\right)$ of Proposition 6.3 , we find that all the Cousin cohomologies are finitely generated. Furthermore, by $\left(B_{l}\right)$ of the same proposition, we obtain

$$
\bigcap_{l} \overline{Z^{l}(M)} \subset \bigcap_{l} U^{l+2}(M)=\emptyset \text {. }
$$

Since Spec $A$ is quasi-compact and $\overline{Z^{-1}(M)} \supset \overline{Z^{0}(M)} \supset \cdots$ is a descending chain of closed subsets, we have $Z^{l}(M)=\emptyset$ for sufficiently large $l$. The proof of Theorem 1.1 is completed.

Corollary 6.4. Let $A$ be a Noetherian ring satisfying (C1)-(C3). For a finitely generated A-module $M$ satisfying (QU), let $\mathfrak{a}^{\prime}(M)$ be the product of all the annihilators of the non-zero Cousin cohomologies of $M$. Then $V\left(\mathfrak{a}^{\prime}(M)\right)$ is the nonCohen-Macaulay locus of $M$. In particular, $\mathrm{ht}_{M} \mathfrak{a}^{\prime}(M)>0$.

Proof. Since only finitely many Cousin cohomologies of $M$ are non-zero, $\mathfrak{a}^{\prime}(M)$ is well defined. Since $H^{p}\left(M^{\bullet}\right)$ is finitely generated, $\operatorname{Supp} H^{p}\left(M^{\bullet}\right)=V\left(\operatorname{ann} H^{p}\left(M^{\bullet}\right)\right)$. Therefore $V\left(\mathfrak{a}^{\prime}(M)\right)=\bigcup H^{p}\left(M^{\bullet}\right)$ is the non-Cohen-Macaulay locus of $M$.

\section{Lemmas FOR TheOREM 1.2}

We prove three lemmas for Theorem 1.2 in this section. They are variants of Theorem 3.6, Corollaries 3.7 and 3.8 of [15], respectively.

Lemma 7.1. Let $x_{1}, \ldots, x_{d} \in A$ be a p-standard sequence on an A-module $M$. We put $\mathfrak{q}_{i}=\left(x_{i}, \ldots, x_{d}\right)$ for all $1 \leq i \leq d$. Then, for any integers $1 \leq j \leq d$ and $n_{1}, \ldots, n_{j} \geq 0$, the following statements hold:

$\left(A_{j}\right)$ If $1 \leq k \leq j$ and $n_{k}>0$, then

$$
\left(x_{k}, \ldots, x_{l-1}\right) M: x_{l} \cap \mathfrak{q}_{1}^{n_{1}} \cdots \mathfrak{q}_{j}^{n_{j}} M=\left(x_{k}, \ldots, x_{l-1}\right) \mathfrak{q}_{1}^{n_{1}} \cdots \mathfrak{q}_{k}^{n_{k}-1} \cdots \mathfrak{q}_{j}^{n_{j}} M
$$

for an arbitrary integer $k \leq l \leq d$.

$\left(B_{j}\right)$ If $2 \leq k \leq j+1 \leq d$ and $n_{k}>0$, then

$$
\left(x_{k}, \ldots, x_{l}\right) \mathfrak{q}_{2}^{n_{2}} \cdots \mathfrak{q}_{j+1}^{n_{j+1}} M: x_{1}=\left(x_{k}, \ldots, x_{l}\right)\left[\mathfrak{q}_{2}^{n_{2}} \cdots \mathfrak{q}_{j+1}^{n_{j+1}} M: x_{1}\right]+0 \underset{\dot{M}}{:} x_{1}
$$

for an arbitrary integer $k \leq l \leq d$. In particular, by letting $l=d$, we have

$$
\mathfrak{q}_{2}^{n_{2}} \cdots \mathfrak{q}_{k}^{n_{k}+1} \cdots \mathfrak{q}_{j+1}^{n_{j+1}} M: x_{1}=\mathfrak{q}_{k}\left[\mathfrak{q}_{2}^{n_{2}} \cdots \mathfrak{q}_{j+1}^{n_{j+1}} M: x_{1}\right]+0 \underset{\dot{M}}{:} x_{1} .
$$

$\left(C_{j}\right)$ If $j<d$ and $n_{2}>0$, then

$$
\mathfrak{q}_{2}^{n_{2}} \cdots \mathfrak{q}_{j+1}^{n_{i+1}} M: x_{1} \subset \mathfrak{q}_{2}^{n_{2}-1} \mathfrak{q}_{3}^{n_{3}} \cdots \mathfrak{q}_{j+1}^{n_{j+1}} M+0 \underset{\dot{M}}{\vdots} x_{1} .
$$

$\left(D_{j}\right)$ If $j<d$ and $n_{2}>0$, then

$$
\mathfrak{q}_{2}^{n_{2}} \cdots \mathfrak{q}_{j+1}^{n_{j+1}} M: x_{1} \cap x_{2} M=x_{2}\left[\mathfrak{q}_{2}^{n_{2}-1} \mathfrak{q}_{3}^{n_{3}} \cdots \mathfrak{q}_{j+1}^{n_{j+1}} M: x_{1}\right] .
$$


$\left(E_{j}\right)$ If $j \leq d-1$ and $n_{1}>1$, then

$$
\mathfrak{q}_{2}^{n_{2}} \cdots \mathfrak{q}_{j+1}^{n_{j+1}} M: x_{1}^{n_{1}}=\mathfrak{q}_{2}^{n_{2}} \cdots \mathfrak{q}_{j+1}^{n_{j+1}} M: x_{1} .
$$

$\left(F_{j}\right)$ If $j \leq d-2$, then

$$
\mathfrak{q}_{3}^{n_{3}} \cdots \mathfrak{q}_{j+2}^{n_{j+2}} M: x_{1} x_{2}=\mathfrak{q}_{3}^{n_{3}} \cdots \mathfrak{q}_{j+2}^{n_{j+2}} M: x_{2} .
$$

Proof. We work by induction on $j$.

$\left(A_{1}\right)$ : Since $x_{1}, \ldots, x_{d}$ is a $d$-sequence on $M$, we obtain (7.1.1). See [9, Theorem 1.3] or [14, Lemma 2.2].

$\left(B_{1}\right)$ : We work by induction on $l$. Let $a$ be an element of the left hand side of (7.1.2). If $l=2$, then we put $x_{1} a=x_{2} b$ where $b \in \mathfrak{q}_{2}^{n_{2}} M$. Since $x_{2}, \ldots, x_{d}$ is a p-standard sequence on $M / x_{1} M$, we have

$$
\begin{aligned}
b & \in x_{1} M: x_{2} \cap\left[x_{1} M+\mathfrak{q}_{2}^{n_{2}} M\right] \\
& =x_{1} M
\end{aligned}
$$

because of $\left(A_{1}\right)$. Let $b=x_{1} a^{\prime}$ where $a^{\prime} \in M$. Then $a^{\prime} \in \mathfrak{q}_{2}^{n_{2}} M: x_{1}$ and $x_{1} a=$ $x_{1} x_{2} a^{\prime}$. Therefore $a \in x_{2}\left[\mathfrak{q}_{2}^{n_{2}} M: x_{1}\right]+0:_{M} x_{1}$.

Next we assume that $l>2$, make the obvious inductive assumption and put $x_{1} a=x_{l} b+c$ where $b \in \mathfrak{q}_{2}^{n_{2}} M, c \in\left(x_{2}, \ldots, x_{l-1}\right) \mathfrak{q}_{2}^{n_{2}} M$. By applying $\left(A_{1}\right)$ to the p-standard sequence $x_{2}, \ldots, x_{d}$ on $M / x_{1} M$, we have

$$
\begin{aligned}
b & \in\left(x_{1}, x_{2}, \ldots, x_{l-1}\right) M: x_{l} \cap\left[x_{1} M+\mathfrak{q}_{2}^{n_{2}} M\right] \\
& =x_{1} M+\left(x_{2}, \ldots, x_{l-1}\right) \mathfrak{q}_{2}^{n_{2}-1} M .
\end{aligned}
$$

Let $b=x_{1} a^{\prime}+c^{\prime}$ where $a^{\prime} \in M$ and $c^{\prime} \in\left(x_{2}, \ldots, x_{l-1}\right) \mathfrak{q}_{2}^{n_{2}-1} M$. Then $a^{\prime} \in \mathfrak{q}_{2}^{n_{2}} M: x_{1}$ and

$$
\begin{aligned}
a-x_{l} a^{\prime} & \in\left(x_{2}, \ldots, x_{l-1}\right) \mathfrak{q}_{2}^{n_{2}} M: x_{1} \\
& =\left(x_{2}, \ldots, x_{l-1}\right)\left[\mathfrak{q}_{2}^{n_{2}} M: x_{1}\right]+0 \underset{\dot{M}}{\dot{:}} x_{1} .
\end{aligned}
$$

Therefore $a \in\left(x_{2}, \ldots, x_{l}\right)\left[\mathfrak{q}_{2}^{n_{2}} M: x_{1}\right]+0:_{M} x_{1}$. The opposite inclusion is obvious.

$\left(C_{1}\right)$ : By using $\left(B_{1}\right)$ repeatedly, we have

$$
\begin{aligned}
\mathfrak{q}_{2}^{n_{2}} M: x_{1} & =\mathfrak{q}_{2}^{n_{2}-1}\left[\mathfrak{q}_{2} M: x_{1}\right]+0 \underset{\dot{M}}{:} x_{1} \\
& \subset \mathfrak{q}_{2}^{n_{2}-1} M+0 \underset{M}{:} x_{1} .
\end{aligned}
$$

$\left(D_{1}\right)$ : If $n_{2}=1$, then both sides of (7.1.4) coincide with $x_{2} M$. We assume that $n_{2}>1$. If $a \in \mathfrak{q}_{2}^{n_{2}} M: x_{1} \cap x_{2} M$, then we obtain

$$
\begin{aligned}
x_{1} a & \in \mathfrak{q}_{2}^{n_{2}} M \cap x_{2} M \\
& =x_{2} \mathfrak{q}_{2}^{n_{2}-1} M
\end{aligned}
$$

by applying $\left(A_{1}\right)$ to the p-standard sequence $x_{2}, \ldots, x_{d}$ on $M$. Therefore

$$
\begin{aligned}
a & \in\left[x_{2} \mathfrak{q}_{2}^{n_{2}-1} M: x_{1}\right] \cap \mathfrak{q}_{1} M \\
& =x_{2}\left[\mathfrak{q}_{2}^{n_{2}-1} M: x_{1}\right]+0 \underset{M}{:} x_{1} \cap \mathfrak{q}_{1} M \\
& =x_{2}\left[\mathfrak{q}_{2}^{n_{2}-1} M: x_{1}\right] .
\end{aligned}
$$

Here we used $\left(B_{1}\right)$ and $\left(A_{1}\right)$ again. The opposite inclusion is obvious.

$\left(E_{1}\right)$ : Since $x_{1}^{n_{1}}, x_{2}, \ldots, x_{d}$ is a p-standard sequence on $M$, we obtain

$$
\mathfrak{q}_{2}^{n_{2}} M: x_{1}^{n_{1}}=\mathfrak{q}_{2}^{n_{2}-1}\left[\mathfrak{q}_{2} M: x_{1}^{n_{1}}\right]+0 \underset{\dot{M}}{:} x_{1}^{n_{1}}
$$


by using $\left(B_{1}\right)$. Since $x_{1}, \ldots, x_{d}$ is a p-standard sequence on $M, \mathfrak{q}_{2} M: x_{1}^{n_{1}}=\mathfrak{q}_{2}: x_{1}$ and $0::_{M} x_{1}^{n_{1}}=0:_{M} x_{1}$.

$\left(F_{1}\right)$ : Let $a \in \mathfrak{q}_{3}^{n_{3}} M: x_{1} x_{2}$. Since $x_{1}, x_{3}, \ldots, x_{d}$ is a p-standard sequence on $M$, we obtain that

$$
\begin{aligned}
x_{2} a & \in \mathfrak{q}_{3}^{n_{3}} M: x_{1} \\
& =\mathfrak{q}_{3}^{n_{3}-1}\left[\mathfrak{q}_{3} M: x_{1}\right]+0 \underset{\dot{M}}{:} x_{1} \\
& \subset \mathfrak{q}_{3}^{n_{3}-1}\left[\mathfrak{q}_{3} M: x_{2}\right]+0 \underset{M}{:} x_{2} .
\end{aligned}
$$

Indeed, since $x_{1}, \ldots, x_{d}$ is a p-standard sequence on $M$,

$$
\begin{gathered}
\mathfrak{q}_{3} M: x_{1} \subset \mathfrak{q}_{3} M: x_{1} x_{2}=\mathfrak{q}_{3} M: x_{2}, \\
0 \underset{\dot{M}}{:} x_{1} \subset 0_{\dot{M}}^{:} x_{1} x_{2}=0 \underset{\dot{M}}{:} x_{2} .
\end{gathered}
$$

Therefore $x_{2}^{2} a \in \mathfrak{q}_{3}^{n_{3}} M$ and hence $a \in \mathfrak{q}_{3}^{n_{3}} M: x_{2}^{2}=\mathfrak{q}_{3}^{n_{3}} M: x_{2}$. Here we apply $\left(E_{1}\right)$ to the p-standard sequence $x_{2}, \ldots, x_{d}$ on $M$.

Next we assume that $j>1$ and that $\left(A_{i}\right)-\left(F_{i}\right)$ are true if $i<j$. We want to prove $\left(A_{j}\right)-\left(F_{j}\right)$.

$\left(A_{j}\right)$ : Since $x_{k}, \ldots, x_{d}$ is a p-standard sequence on $M, 0:_{M} x_{k} \cap \mathfrak{q}_{k} M=0$. If $l=k$, then it follows that (7.1.1) holds because $n_{k}>0$.

Assume that $k<l$ and let $a$ be an element of the left hand side of (7.1.1). If $k=1$, then we obtain

$$
\begin{aligned}
a & \in\left(x_{1}, \ldots, x_{l-1}\right) M: x_{l} \cap\left[x_{1} M+\mathfrak{q}_{2}^{n_{1}+n_{2}} \mathfrak{q}_{3}^{n_{3}} \cdots \mathfrak{q}_{j}^{n_{j}} M\right] \\
& =x_{1} M+\left(x_{2}, \ldots, x_{l-1}\right) \mathfrak{q}_{2}^{n_{1}+n_{2}-1} \mathfrak{q}_{3}^{n_{3}} \cdots \mathfrak{q}_{j}^{n_{j}} M
\end{aligned}
$$

by using $\left(A_{j-1}\right)$. Indeed, $x_{2}, \ldots, x_{d}$ is a p-standard sequence on $M / x_{1} M$ and $n_{1}>0$ by the assumption. Since

$$
a \in \mathfrak{q}_{1}^{n_{1}} \cdots \mathfrak{q}_{j}^{n_{j}} M=x_{1} \mathfrak{q}_{1}^{n_{1}-1} \mathfrak{q}_{2}^{n_{2}} \cdots \mathfrak{q}_{j}^{n_{j}} M+\mathfrak{q}_{2}^{n_{1}+n_{2}} \mathfrak{q}_{3}^{n_{3}} \cdots \mathfrak{q}_{j}^{n_{j}} M,
$$

we obtain

$$
\begin{aligned}
a \in & x_{1} \mathfrak{q}_{1}^{n_{1}-1} \mathfrak{q}_{2}^{n_{2}} \cdots \mathfrak{q}_{j}^{n_{j}} M \\
& +\left(x_{2}, \ldots, x_{l-1}\right) \mathfrak{q}_{2}^{n_{1}+n_{2}-1} \mathfrak{q}_{3}^{n_{3}} \cdots \mathfrak{q}_{j}^{n_{j}} M \\
& +x_{1} M \cap \mathfrak{q}_{2}^{n_{1}+n_{2}} \mathfrak{q}_{3}^{n_{3}} \cdots \mathfrak{q}_{j}^{n_{j}} M .
\end{aligned}
$$

By using $\left(C_{j-1}\right)$, we have

$$
\begin{aligned}
x_{1} M \cap \mathfrak{q}_{2}^{n_{1}+n_{2}} \mathfrak{q}_{3}^{n_{3}} \cdots \mathfrak{q}_{j}^{n_{j}} M & =x_{1}\left[\mathfrak{q}_{2}^{n_{1}+n_{2}} \mathfrak{q}_{3}^{n_{3}} \cdots \mathfrak{q}_{j}^{n_{j}} M: x_{1}\right] \\
& \subset x_{1} \mathfrak{q}_{2}^{n_{1}+n_{2}-1} \mathfrak{q}_{3}^{n_{3}} \cdots \mathfrak{q}_{j}^{n_{j}} M .
\end{aligned}
$$

Therefore

$$
a \in\left(x_{1}, \ldots, x_{l-1}\right) \mathfrak{q}_{1}^{n_{1}-1} \mathfrak{q}_{2}^{n_{2}} \cdots \mathfrak{q}_{j}^{n_{j}} M
$$

If $k>1$, then we work by induction on $n_{1}$. If $n_{1}=0$, then

$$
\left(x_{k}, \ldots, x_{l-1}\right) M: x_{l} \cap \mathfrak{q}_{2}^{n_{2}} \cdots \mathfrak{q}_{j}^{n_{j}} M=\left(x_{k}, \ldots, x_{l-1}\right) \mathfrak{q}_{2}^{n_{2}} \cdots \mathfrak{q}_{k}^{n_{k}-1} \cdots \mathfrak{q}_{j}^{n_{j}} M
$$

because of $\left(A_{j-1}\right)$. Assume that $n_{1}>0$ and make the obvious inductive assumption. Since $x_{2}, \ldots, x_{d}$ is a p-standard sequence on $M / x_{1} M$,

$$
\begin{aligned}
a & \in\left(x_{1}, x_{k}, \ldots, x_{l-1}\right) M: x_{l} \cap\left[x_{1} M+\mathfrak{q}_{2}^{n_{1}+n_{2}} \mathfrak{q}_{3}^{n_{3}} \cdots \mathfrak{q}_{j}^{n_{j}} M\right] \\
& =x_{1} M+\left(x_{k}, \ldots, x_{l-1}\right) \mathfrak{q}_{2}^{n_{1}+n_{2}} \mathfrak{q}_{3}^{n_{3}} \cdots \mathfrak{q}_{k}^{n_{k}-1} \cdots \mathfrak{q}_{j}^{n_{j}} M .
\end{aligned}
$$


By using (7.1.7) and $\left(C_{j-1}\right)$ we have

$$
\begin{aligned}
a \in & x_{1} \mathfrak{q}_{1}^{n_{1}-1} \mathfrak{q}_{2}^{n_{2}} \cdots \mathfrak{q}_{j}^{n_{j}} M \\
& +\left(x_{k}, \ldots, x_{l-1}\right) \mathfrak{q}_{2}^{n_{1}+n_{2}} \mathfrak{q}_{3}^{n_{3}} \cdots \mathfrak{q}_{k}^{n_{k}-1} \cdots \mathfrak{q}_{j}^{n_{j}} M \\
& +x_{1}\left[\mathfrak{q}_{2}^{n_{1}+n_{2}} \mathfrak{q}_{3}^{n_{3}} \cdots \mathfrak{q}_{j}^{n_{j}} M: x_{1}\right] \\
\subset & x_{1} \mathfrak{q}_{1}^{n_{1}-1} \mathfrak{q}_{2}^{n_{2}} \cdots \mathfrak{q}_{j}^{n_{j}} M \\
& +\left(x_{k}, \ldots, x_{l-1}\right) \mathfrak{q}_{2}^{n_{1}+n_{2}} \mathfrak{q}_{3}^{n_{3}} \cdots \mathfrak{q}_{k}^{n_{k}-1} \ldots \mathfrak{q}_{j}^{n_{j}} M .
\end{aligned}
$$

Let $a=x_{1} a^{\prime}+b$ where $a^{\prime} \in \mathfrak{q}_{1}^{n_{1}-1} \cdots \mathfrak{q}_{j}^{n_{j}} M$ and

$$
b \in\left(x_{k}, \ldots, x_{l-1}\right) \mathfrak{q}_{2}^{n_{1}+n_{2}} \mathfrak{q}_{3}^{n_{3}} \cdots \mathfrak{q}_{k}^{n_{k}-1} \cdots \mathfrak{q}_{j}^{n_{j}} M .
$$

Then

$$
\begin{aligned}
a^{\prime} & \in\left(x_{k}, \ldots, x_{l-1}\right) M: x_{1} x_{l} \cap \mathfrak{q}_{1}^{n_{1}-1} \cdots \mathfrak{q}_{j}^{n_{j}} M \\
& =\left(x_{k}, \ldots, x_{l-1}\right) M: x_{l} \cap \mathfrak{q}_{1}^{n_{1}-1} \cdots \mathfrak{q}_{j}^{n_{j}} M \\
& =\left(x_{k}, \ldots, x_{l-1}\right) \mathfrak{q}_{1}^{n_{1}-1} \cdots \mathfrak{q}_{k}^{n_{k}-1} \cdots \mathfrak{q}_{j}^{n_{j}} M .
\end{aligned}
$$

Here we used the inductive assumption. Thus the left hand side of (7.1.1) is contained in the right hand side. The opposite inclusion is obvious.

$\left(B_{j}\right)$ : We work by induction on $l$. Let $a$ be an element of the left hand side of (7.1.2). If $l=k$, then we put $x_{1} a=x_{k} b$ where $b \in \mathfrak{q}_{2}^{n_{2}} \cdots \mathfrak{q}_{j+1}^{n_{j+1}} M$. By applying $\left(A_{j-1}\right)$ to the p-standard sequence $x_{2}, \ldots, x_{d}$ on $M / x_{1} M$, we obtain

$$
b \in x_{1} M: x_{k} \cap\left[x_{1} M+\mathfrak{q}_{2}^{n_{2}} \cdots \mathfrak{q}_{j+1}^{n_{j+1}} M\right]=x_{1} M .
$$

Let $b=x_{1} a^{\prime}$. Then $a^{\prime} \in \mathfrak{q}_{2}^{n_{2}} \cdots \mathfrak{q}_{j+1}^{n_{j+1}} M: x_{1}$ and $a \in x_{k}\left[\mathfrak{q}_{2}^{n_{2}} \cdots \mathfrak{q}_{j+1}^{n_{j+1}} M: x_{1}\right]+$ $0:_{M} x_{1}$.

Assume that $l>k$ and make the obvious inductive assumption. Let $x_{1} a=$ $x_{l} b+c$ where $b \in \mathfrak{q}_{2}^{n_{2}} \ldots \mathfrak{q}_{j+1}^{n_{j+1}} M$ and $c \in\left(x_{k}, \ldots, x_{l-1}\right) \mathfrak{q}_{2}^{n_{2}} \cdots \mathfrak{q}_{j+1}^{n_{j+1}} M$. Then we obtain

$$
\begin{aligned}
b & \in\left(x_{1}, x_{k}, \ldots, x_{l-1}\right) M: x_{l} \cap\left[x_{1} M+\mathfrak{q}_{2}^{n_{2}} \cdots \mathfrak{q}_{j+1}^{n_{j+1}} M\right] \\
& =x_{1} M+\left(x_{k}, \ldots, x_{l-1}\right) \mathfrak{q}_{2}^{n_{2}} \cdots \mathfrak{q}_{k}^{n_{k}-1} \cdots \mathfrak{q}_{j+1}^{n_{j+1}} M
\end{aligned}
$$

by using $\left(A_{j-1}\right)$. Let $b=x_{1} a^{\prime}+c^{\prime}$ where $a^{\prime} \in M$ and

$$
c^{\prime} \in\left(x_{k}, \ldots, x_{l-1}\right) \mathfrak{q}_{2}^{n_{2}} \cdots \mathfrak{q}_{k}^{n_{k}-1} \cdots \mathfrak{q}_{j+1}^{n_{j+1}} M .
$$

Then $a^{\prime} \in \mathfrak{q}_{2}^{n_{2}} \cdots \mathfrak{q}_{j+1}^{n_{j+1}} M: x_{1}$ and

$$
\begin{aligned}
a-x_{l} a^{\prime} & \in\left(x_{k}, \ldots, x_{l-1}\right) \mathfrak{q}_{2}^{n_{2}} \cdots \mathfrak{q}_{j+1}^{n_{j+1}} M: x_{1} \\
& =\left(x_{k}, \ldots, x_{l-1}\right)\left[\mathfrak{q}_{2}^{n_{2}} \cdots \mathfrak{q}_{j+1}^{n_{j+1}} M: x_{1}\right]+0 \underset{M}{:} x_{1} .
\end{aligned}
$$

Therefore $a$ is in the right hand side of (7.1.2). The opposite inclusion is obvious.

$\left(C_{j}\right)$ : By using $\left(B_{j}\right)$, we may assume that $n_{2}=1$. If $n_{j+1}=0$, then the inclusion comes from $\left(C_{j-1}\right)$. We may also assume that $n_{j+1}>0$.

Let $a$ be an element of the left hand side of (7.1.3). By applying $\left(C_{j-1}\right)$ to the p-standard sequence $x_{1}, x_{3}, \ldots, x_{d}$ on $M / x_{2} M$, we obtain

$$
\begin{aligned}
a & \in\left[x_{2} M+\mathfrak{q}_{3}^{n_{3}+1} \cdots \mathfrak{q}_{j+1}^{n_{j+1}} M\right]: x_{1} \\
& \subset \mathfrak{q}_{3}^{n_{3}} \cdots \mathfrak{q}_{j+1}^{n_{j+1}} M+x_{2} M: x_{1} .
\end{aligned}
$$


On the other hand, since $n_{j+1}>0$,

$$
a \in \mathfrak{q}_{2}^{2} M: x_{1} \subset \mathfrak{q}_{2} M+0: x_{1} .
$$

Here we used $\left(C_{1}\right)$. Therefore

$$
\begin{aligned}
a & \in \mathfrak{q}_{3}^{n_{3}} \cdots \mathfrak{q}_{j+1}^{n_{j+1}} M+0 \underset{\dot{M}}{:} x_{1}+x_{2} M: x_{1} \cap \mathfrak{q}_{2} M \\
& \subset \mathfrak{q}_{3}^{n_{3}} \cdots \mathfrak{q}_{j+1}^{n_{j+1}} M+0 \underset{\dot{M}}{:} x_{1}+x_{2} M: x_{3} \cap \mathfrak{q}_{2} M \\
& =\mathfrak{q}_{3}^{n_{3}} \cdots \mathfrak{q}_{j+1}^{n_{j+1}} M+0 \underset{\dot{M}}{\dot{:}} x_{1}+x_{2} M .
\end{aligned}
$$

Since $a \in \mathfrak{q}_{3}^{n_{3}} \cdots \mathfrak{q}_{j+1}^{n_{j+1}} M: x_{1}$,

$$
\begin{aligned}
a & \in \mathfrak{q}_{3}^{n_{3}} \cdots \mathfrak{q}_{j+1}^{n_{j+1}} M+0 \underset{M}{:} x_{1}+x_{2} M \cap\left[\mathfrak{q}_{3}^{n_{3}} \cdots \mathfrak{q}_{j+1}^{n_{j+1}} M: x_{1}\right] \\
& =\mathfrak{q}_{3}^{n_{3}} \cdots \mathfrak{q}_{j+1}^{n_{j+1}} M+0_{\dot{M}}^{:} x_{1}+x_{2}\left[\mathfrak{q}_{3}^{n_{3}} \cdots \mathfrak{q}_{j+1}^{n_{j+1}} M: x_{1} x_{2}\right] \\
& =\mathfrak{q}_{3}^{n_{3}} \cdots \mathfrak{q}_{j+1}^{n_{j+1}} M+0 \underset{M}{:} x_{1}+x_{2}\left[\mathfrak{q}_{3}^{n_{3}} \cdots \mathfrak{q}_{j+1}^{n_{j+1}} M: x_{2}\right] \\
& =\mathfrak{q}_{3}^{n_{3}} \cdots \mathfrak{q}_{j+1}^{n_{j+1}} M+0 \underset{\dot{M}}{\vdots} x_{1} .
\end{aligned}
$$

Here we used $\left(F_{j-1}\right)$.

$\left(D_{j}\right)$ : If $n_{j+1}=0$, then the equation follows from $\left(D_{j-1}\right)$. Thus we may assume that $n_{j+1}>0$. Let $a$ be an element of the left hand side of (7.1.4). Then

$$
\begin{aligned}
x_{1} a & \in x_{2} M \cap \mathfrak{q}_{2}^{n_{2}} \cdots \mathfrak{q}_{j+1}^{n_{j+1}} M \\
& =x_{2} \mathfrak{q}_{2}^{n_{2}-1} \cdots \mathfrak{q}_{j+1}^{n_{j+1}} M,
\end{aligned}
$$

where we used $\left(A_{j}\right)$. Let $x_{1} a=x_{2} b$ where $b \in \mathfrak{q}_{2}^{n_{2}-1} \cdots \mathfrak{q}_{j+1}^{n_{j+1}} M$. Then

$$
b \in x_{1} M: x_{2} \cap \mathfrak{q}_{j+1} M \subset x_{1} M: x_{2} \cap \mathfrak{q}_{1} M=x_{1} M .
$$

Let $b=x_{1} a^{\prime}$. Then $a^{\prime} \in \mathfrak{q}_{2}^{n_{2}-1} \cdots \mathfrak{q}_{j+1}^{n_{j+1}} M: x_{1}$ and $a-x_{2} a^{\prime} \in 0::_{M} x_{1} \cap x_{2} M \subset$ $0: M x_{1} \cap \mathfrak{q}_{1} M=0$. That is, $a=x_{2} a^{\prime} \in x_{2}\left[\mathfrak{q}_{2}^{n_{2}-1} \cdots \mathfrak{q}_{j+1}^{n_{j+1}} M: x_{1}\right]$. The opposite inclusion is obvious.

$\left(E_{j}\right)$ : We may assume that $n_{1}=2$. Furthermore we may also assume that $n_{2}=1$ and $n_{j+1}>0$ because of $\left(B_{j}\right)$ and $\left(E_{j-1}\right)$.

First we assume that $n_{3}+\cdots+n_{j+1}=1$; that is, $n_{3}=\cdots=n_{j}=0$ and $n_{j+1}=1$. We show that

$$
\left(x_{2}, \ldots, x_{l}, x_{j+1}, \ldots, x_{d}\right) \mathfrak{q}_{2} M: x_{1}^{2}=\left(x_{2}, \ldots, x_{l}, x_{j+1}, \ldots, x_{d}\right) \mathfrak{q}_{2} M: x_{1}
$$

by descending induction on $l$. If $l=j$, then the equation comes from $\left(E_{1}\right)$. Assume that $l<j$, make the obvious inductive assumption and let $a$ be an element of the left hand side of the equation. Then

$$
\begin{aligned}
a & \in\left(x_{2}, \ldots, x_{l+1}, x_{j+1}, \ldots, x_{d}\right) \mathfrak{q}_{2} M: x_{1}^{2} \\
& =\left(x_{2}, \ldots, x_{l+1}, x_{j+1}, \ldots, x_{d}\right) \mathfrak{q}_{2} M: x_{1} .
\end{aligned}
$$

Let $x_{1} a=x_{l+1} b+c$ where $b \in \mathfrak{q}_{2} M$ and $c \in\left(x_{2}, \ldots, x_{l}, x_{j+1}, \ldots, x_{d}\right) \mathfrak{q}_{2} M$. Then

$$
\begin{aligned}
b & \in\left(x_{2}, \ldots, x_{l}, x_{j+1}, \ldots, x_{d}\right) M: x_{1} x_{l+1} \cap \mathfrak{q}_{2} M \\
& =\left(x_{2}, \ldots, x_{l}, x_{j+1}, \ldots, x_{d}\right) M: x_{l+1} \cap \mathfrak{q}_{2} M \\
& =\left(x_{2}, \ldots, x_{l}, x_{j+1}, \ldots, x_{d}\right) M .
\end{aligned}
$$


Here we apply $\left(A_{1}\right)$ to the p-standard sequence $x_{2}, \ldots, x_{j}$ on $M /\left(x_{j+1}, \ldots, x_{d}\right) M$. Thus $x_{1} a \in\left(x_{2}, \ldots, x_{l}, x_{j+1}, \ldots, x_{d}\right) \mathfrak{q}_{2} M$. If we put $l=1$, then we have

$$
\mathfrak{q}_{2} \mathfrak{q}_{j+1} M: x_{1}^{2}=\mathfrak{q}_{2} \mathfrak{q}_{j+1} M: x_{1} .
$$

Next we assume that $n_{3}+\cdots+n_{j+1}>1$ and let $a$ be an element of the left hand side of (7.1.5). By applying $\left(E_{j-1}\right)$ to the p-standard sequence $x_{1}, x_{3}, \ldots, x_{d}$ on $M / x_{2} M$, we have

$$
\begin{aligned}
a & \in\left[x_{2} M+\mathfrak{q}_{3}^{n_{3}+1} \cdots \mathfrak{q}_{j+1}^{n_{j+1}} M\right]: x_{1}^{2} \\
& =\left[x_{2} M+\mathfrak{q}_{3}^{n_{3}+1} \cdots \mathfrak{q}_{j+1}^{n_{j+1}} M\right]: x_{1} .
\end{aligned}
$$

Therefore

$$
\begin{aligned}
x_{1} a & \in\left[x_{2} M+\mathfrak{q}_{3}^{n_{3}+1} \cdots \mathfrak{q}_{j+1}^{n_{j+1}} M\right] \cap\left[\mathfrak{q}_{2} \mathfrak{q}_{3}^{n_{3}} \cdots \mathfrak{q}_{j+1}^{n_{j+1}} M: x_{1}\right] \\
& =\mathfrak{q}_{3}^{n_{3}+1} \cdots \mathfrak{q}_{j+1}^{n_{j+1}} M+x_{2} M \cap\left[\mathfrak{q}_{2} \mathfrak{q}_{3}^{n_{3}} \cdots \mathfrak{q}_{j+1}^{n_{j+1}} M: x_{1}\right] \\
& =\mathfrak{q}_{3}^{n_{3}+1} \cdots \mathfrak{q}_{j+1}^{n_{j+1}} M+x_{2}\left[\mathfrak{q}_{3}^{n_{3}} \cdots \mathfrak{q}_{j+1}^{n_{j+1}} M: x_{1}\right] .
\end{aligned}
$$

Here we used $\left(D_{j}\right)$. Let $x_{1} a=x_{2} b+c$ where $b \in \mathfrak{q}_{3}^{n_{3}} \cdots \mathfrak{q}_{j+1}^{n_{j+1}} M: x_{1}$ and $c \in$ $\mathfrak{q}_{3}^{n_{3}+1} \cdots \mathfrak{q}_{j+1}^{n_{j+1}} M$. Then

$$
\begin{aligned}
b & \in\left[x_{1} M+\mathfrak{q}_{3}^{n_{3}+1} \cdots \mathfrak{q}_{j+1}^{n_{j+1}} M\right]: x_{2} \\
& \subset x_{1} M: x_{2}+\mathfrak{q}_{3}^{n_{3}} \cdots \mathfrak{q}_{j+1}^{n_{j+1}} M
\end{aligned}
$$

because of $\left(C_{j-1}\right)$. On the other hand, since $n_{3}+\cdots+n_{j+1}>1, b \in \mathfrak{q}_{3}^{2} M: x_{1} \subset$ $\mathfrak{q}_{3} M+0:_{M} x_{1}$. Therefore

$$
\begin{aligned}
b & \in \mathfrak{q}_{3}^{n_{3}} \cdots \mathfrak{q}_{j+1}^{n_{j+1}} M+0 \underset{M}{:} x_{1}+x_{1} M: x_{2} \cap \mathfrak{q}_{3} M \\
& \subset \mathfrak{q}_{3}^{n_{3}} \cdots \mathfrak{q}_{j+1}^{n_{j+1}} M+0 \underset{\dot{M}}{:} x_{1}+x_{1} M,
\end{aligned}
$$

because $x_{1} M: x_{2} \cap \mathfrak{q}_{3} M \subset x_{1} M: x_{2} \cap \mathfrak{q}_{1} M=x_{1} M$. Since $b \in \mathfrak{q}_{3}^{n_{3}} \cdots \mathfrak{q}_{j+1}^{n_{j+1}} M: x_{1}$, we obtain

$$
\begin{aligned}
b & \in \mathfrak{q}_{3}^{n_{3}} \cdots \mathfrak{q}_{j+1}^{n_{j+1}} M+0 \underset{M}{:} x_{1}+x_{1} M \cap\left[\mathfrak{q}_{3}^{n_{3}} \cdots \mathfrak{q}_{j+1}^{n_{j+1}} M: x_{1}\right] \\
& =\mathfrak{q}_{3}^{n_{3}} \cdots \mathfrak{q}_{j+1}^{n_{j+1}} M+0_{\dot{M}}^{:} x_{1}+x_{1}\left[\mathfrak{q}_{3}^{n_{3}} \cdots \mathfrak{q}_{j+1}^{n_{j+1}} M: x_{1}^{2}\right] \\
& =\mathfrak{q}_{3}^{n_{3}} \cdots \mathfrak{q}_{j+1}^{n_{j+1}} M+0_{\dot{M}}^{:} x_{1} .
\end{aligned}
$$

Here we used $\left(E_{j-1}\right)$. Thus $x_{1} a=x_{2} b+c \in \mathfrak{q}_{2} \mathfrak{q}_{3}^{n_{3}} \cdots \mathfrak{q}_{j+1}^{n_{j+1}} M$.

$\left(F_{j}\right)$ : First we show that $y_{2}=x_{1} x_{2}, y_{3}=x_{3}, \ldots, y_{d}=x_{d}$ is a p-standard sequence on $M$. In other words, we prove

$$
\left(y_{\lambda}^{n_{\lambda}} \mid \lambda \in \Lambda\right) M: y_{i}^{n_{i}} y_{j}^{n_{j}}=\left(y_{\lambda}^{n_{\lambda}} \mid \lambda \in \Lambda\right) M: y_{j}^{n_{j}}
$$

for any positive integers $n_{2}, \ldots, n_{d}$, any subset $\Lambda \subsetneq\{2, \ldots, d\}$ and $i, j \in\{2, \ldots, d\} \backslash$ $\Lambda$ such that $i \leq j$ or $s<j$ (where $s$ is the type of the p-standard sequence $x_{1}, \ldots$, $x_{d}$ on $\left.M\right)$.

If $2 \notin \Lambda$, then (7.1.8) is obvious. Assume that $2 \in \Lambda$ and let $\Lambda^{\prime}=\Lambda \backslash\{2\}$. We divide (7.1.8) into two parts:

$$
\begin{aligned}
\left(y_{\lambda}^{n_{\lambda}} \mid \lambda \in \Lambda\right) M: y_{i}^{n_{i}} & \subset\left(y_{\lambda}^{n_{\lambda}} \mid \lambda \in \Lambda\right) M: y_{j}, \\
\left(y_{\lambda}^{n_{\lambda}} \mid \lambda \in \Lambda\right) M: y_{j}^{n_{j}+1} & =\left(y_{\lambda}^{n_{\lambda}} \mid \lambda \in \Lambda\right) M: y_{j} .
\end{aligned}
$$


If $a$ is an element of the left hand side of (7.1.9), then we put $x_{i}^{n_{i}} a=\left(x_{1} x_{2}\right)^{n_{2}} b+c$ where $b \in M$ and $c \in\left(x_{\lambda}^{n_{\lambda}} \mid \lambda \in \Lambda^{\prime}\right) M$. Since $\Lambda^{\prime} \subset\{3, \ldots, d\}$ and $i \notin \Lambda^{\prime}$, we have

$$
\begin{aligned}
b & \in\left[x_{i}^{n_{i}} M+\left(x_{\lambda}^{n_{\lambda}} \mid \lambda \in \Lambda^{\prime}\right) M\right]: x_{1}^{n_{2}} x_{2}^{n_{2}} \\
& =\left[x_{i}^{n_{i}} M+\left(x_{\lambda}^{n_{\lambda}} \mid \lambda \in \Lambda^{\prime}\right) M\right]: x_{2}^{n_{2}} .
\end{aligned}
$$

If we put $x_{2}^{n_{2}} b=x_{i}^{n_{i}} a^{\prime}+c^{\prime}$ where $a^{\prime} \in M, c^{\prime} \in\left(x_{\lambda}^{n_{\lambda}} \mid \lambda \in \Lambda^{\prime}\right) M$, then $a^{\prime} \in$ $\left[x_{2}^{n_{2}} M+\left(x_{\lambda}^{n_{\lambda}} \mid \lambda \in \Lambda^{\prime}\right) M\right]: x_{i}^{n_{i}}$ and $a-x_{1}^{n_{2}} a^{\prime} \in\left(x_{\lambda}^{n_{\lambda}} \mid \lambda \in \Lambda^{\prime}\right) M: x_{i}^{n_{i}}$. Thus

$$
\begin{aligned}
a \in & x_{1}^{n_{2}}\left[\left[x_{2}^{n_{2}} M+\left(x_{\lambda}^{n_{\lambda}} \mid \lambda \in \Lambda^{\prime}\right) M\right]: x_{i}^{n_{i}}\right] \\
& +\left(x_{\lambda}^{n_{\lambda}} \mid \lambda \in \Lambda^{\prime}\right) M: x_{i}^{n_{i}} \\
\subset & {\left[x_{1}^{n_{2}} x_{2}^{n_{2}} M+\left(x_{\lambda}^{n_{\lambda}} \mid \lambda \in \Lambda^{\prime}\right) M\right]: x_{j}, }
\end{aligned}
$$

because

$$
\begin{aligned}
{\left[x_{2}^{n_{2}} M+\left(x_{\lambda}^{n_{\lambda}} \mid \lambda \in \Lambda^{\prime}\right) M\right]: x_{i}^{n_{i}} } & \subset\left[x_{2}^{n_{2}} M+\left(x_{\lambda}^{n_{\lambda}} \mid \lambda \in \Lambda^{\prime}\right) M\right]: x_{i}^{n_{i}} x_{j} \\
& =\left[x_{2}^{n_{2}} M+\left(x_{\lambda}^{n_{\lambda}} \mid \lambda \in \Lambda^{\prime}\right) M\right]: x_{j}, \\
\left(x_{\lambda}^{n_{\lambda}} \mid \lambda \in \Lambda^{\prime}\right) M: x_{i}^{n_{i}} & \subset\left(x_{\lambda}^{n_{\lambda}} \mid \lambda \in \Lambda^{\prime}\right) M: x_{i}^{n_{i}} x_{j} \\
& =\left(x_{\lambda}^{n_{\lambda}} \mid \lambda \in \Lambda^{\prime}\right) M: x_{j} .
\end{aligned}
$$

Thus we obtain (7.1.9).

In the same way as above, we have

$$
\begin{aligned}
{\left[x_{1}^{n_{2}} x_{2}^{n_{2}} M+\left(x_{\lambda}^{n_{\lambda}} \mid \lambda \in \Lambda^{\prime}\right) M\right]: x_{j}^{n_{j}+1} } & =x_{1}^{n_{2}}\left[\left[x_{2}^{n_{2}} M+\left(x_{\lambda}^{n_{\lambda}} \mid \lambda \in \Lambda^{\prime}\right) M\right]: x_{j}\right] \\
& +\left(x_{\lambda}^{n_{\lambda}} \mid \lambda \in \Lambda^{\prime}\right) M: x_{j} \\
\subset & {\left[x_{1}^{n_{2}} x_{2}^{n_{2}} M+\left(x_{\lambda}^{n_{\lambda}} \mid \lambda \in \Lambda^{\prime}\right) M\right]: x_{j} . }
\end{aligned}
$$

Thus we also obtain (7.1.10).

Therefore we may assume that $n_{3}=1$ on use of $\left(B_{j}\right)$. We may also assume that $n_{j+2}>0$ because of $\left(F_{j-1}\right)$. Assume that $n_{4}+\cdots+n_{j+2}=1$, that is, $n_{4}=\cdots=n_{j+1}=0$ and $n_{j+2}=1$. We show that

$$
\left(x_{3}, \ldots, x_{l}, x_{j+2}, \ldots, x_{d}\right) \mathfrak{q}_{3} M: x_{1} x_{2}=\left(x_{3}, \ldots, x_{l}, x_{j+2}, \ldots, x_{d}\right) \mathfrak{q}_{3} M: x_{2}
$$

by descending induction on $l$. If $l=j+1$, then the equation comes from $\left(F_{1}\right)$. Assume that $l<j+1$, make the obvious inductive assumption and let $a$ be an element of the left hand side of the equation. Then

$$
\begin{aligned}
a & \in\left(x_{3}, \ldots, x_{l+1}, x_{j+2}, \ldots, x_{d}\right) \mathfrak{q}_{3} M: x_{1} x_{2} \\
& =\left(x_{3}, \ldots, x_{l+1}, x_{j+2}, \ldots, x_{d}\right) \mathfrak{q}_{3} M: x_{2} .
\end{aligned}
$$

Let $x_{2} a=x_{l+1} b+c$ where $b \in \mathfrak{q}_{3} M$ and $c \in\left(x_{3}, \ldots, x_{l}, x_{j+2}, \ldots, x_{d}\right) \mathfrak{q}_{3} M$. Then

$$
\begin{aligned}
b & \in\left(x_{3}, \ldots, x_{l}, x_{j+2}, \ldots, x_{d}\right) M: x_{1} x_{l+1} \cap \mathfrak{q}_{3} M \\
& =\left(x_{3}, \ldots, x_{l}, x_{j+2}, \ldots, x_{d}\right) M: x_{l+1} \cap \mathfrak{q}_{3} M \\
& =\left(x_{3}, \ldots, x_{l}, x_{j+2}, \ldots, x_{d}\right) M .
\end{aligned}
$$

Here we apply $\left(A_{1}\right)$ to the p-standard sequence $x_{3}, \ldots, x_{j+1}$ on $M /\left(x_{j+2}, \ldots, x_{d}\right) M$. Thus $x_{2} a \in\left(x_{3}, \ldots, x_{l}, x_{j+2}, \ldots x_{d}\right) \mathfrak{q}_{3} M$. If we put $l=2$, then we have

$$
\mathfrak{q}_{3} \mathfrak{q}_{j+2} M: x_{1} x_{2}=\mathfrak{q}_{3} \mathfrak{q}_{j+2} M: x_{2} .
$$


Next we assume that $n_{4}+\cdots+n_{j+2}>1$ and let $a$ be an element of the left hand side of (7.1.6). By applying $\left(F_{j-1}\right)$ to the p-standard sequence $x_{1}, x_{2}, x_{4}, \ldots, x_{d}$ on $M / x_{3} M$, we have

$$
\begin{aligned}
a & \in\left[x_{3} M+\mathfrak{q}_{4}^{n_{4}+1} \cdots \mathfrak{q}_{j+2}^{n_{j+2}} M\right]: x_{1} x_{2} \\
& =\left[x_{3} M+\mathfrak{q}_{4}^{n_{4}+1} \cdots \mathfrak{q}_{j+2}^{n_{j+2}} M\right]: x_{2} .
\end{aligned}
$$

Therefore

$$
\begin{aligned}
x_{2} a & \in\left[x_{3} M+\mathfrak{q}_{4}^{n_{4}+1} \cdots \mathfrak{q}_{j+2}^{n_{j+2}} M\right] \cap\left[\mathfrak{q}_{3} \mathfrak{q}_{4}^{n_{4}} \cdots \mathfrak{q}_{j+2}^{n_{j+2}} M: x_{1}\right] \\
& =\mathfrak{q}_{4}^{n_{4}+1} \cdots \mathfrak{q}_{j+2}^{n_{j+2}} M+x_{3} M \cap\left[\mathfrak{q}_{3} \mathfrak{q}_{4}^{n_{4}} \cdots \mathfrak{q}_{j+2}^{n_{j+2}} M: x_{1}\right] \\
& =\mathfrak{q}_{4}^{n_{4}+1} \cdots \mathfrak{q}_{j+2}^{n_{j+2}} M+x_{3}\left[\mathfrak{q}_{4}^{n_{4}} \cdots \mathfrak{q}_{j+2}^{n_{j+2}} M: x_{1}\right] .
\end{aligned}
$$

Here we used $\left(D_{j}\right)$. Let $x_{2} a=x_{3} b+c$ where $b \in \mathfrak{q}_{4}^{n_{4}} \cdots \mathfrak{q}_{j+2}^{n_{j+2}} M: x_{1}$ and $c \in$ $\mathfrak{q}_{4}^{n_{4}+1} \cdots \mathfrak{q}_{j+2}^{n_{j+2}} M$. Then

$$
\begin{aligned}
b & \in\left[x_{2} M+\mathfrak{q}_{4}^{n_{4}+1} \cdots \mathfrak{q}_{j+2}^{n_{j+2}} M\right]: x_{3} \\
& \subset x_{2} M: x_{3}+\mathfrak{q}_{4}^{n_{4}} \cdots \mathfrak{q}_{j+2}^{n_{j+2}} M
\end{aligned}
$$

because of $\left(C_{j-1}\right)$. On the other hand, since $n_{4}+\cdots+n_{j+2}>1, b \in \mathfrak{q}_{4}^{2} M: x_{1} \subset$ $\mathfrak{q}_{4} M+0:_{M} x_{1}$. Therefore

$$
\begin{aligned}
b & \in \mathfrak{q}_{4}^{n_{4}} \cdots \mathfrak{q}_{j+2}^{n_{j+2}} M+0 \underset{M}{:} x_{1}+x_{2} M: x_{3} \cap \mathfrak{q}_{4} M \\
& \subset \mathfrak{q}_{4}^{n_{4}} \cdots \mathfrak{q}_{j+2}^{n_{j+2}} M+0 \underset{\dot{M}}{:} x_{1}+x_{2} M,
\end{aligned}
$$

because $x_{2} M: x_{3} \cap \mathfrak{q}_{4} M \subset x_{2} M: x_{3} \cap \mathfrak{q}_{2} M=x_{2} M$. Since $b \in \mathfrak{q}_{4}^{n_{4}} \cdots \mathfrak{q}_{j+2}^{n_{j+2}} M: x_{1}$, we obtain

$$
\begin{aligned}
b & \in \mathfrak{q}_{4}^{n_{4}} \cdots \mathfrak{q}_{j+2}^{n_{j+2}} M+0 \underset{M}{:} x_{1}+x_{2} M \cap\left[\mathfrak{q}_{4}^{n_{4}} \cdots \mathfrak{q}_{j+2}^{n_{j+2}} M: x_{1}\right] \\
& =\mathfrak{q}_{4}^{n_{4}} \cdots \mathfrak{q}_{j+2}^{n_{j+2}} M+0_{\dot{M}}^{:} x_{1}+x_{2}\left[\mathfrak{q}_{4}^{n_{4}} \cdots \mathfrak{q}_{j+2}^{n_{j+2}} M: x_{1} x_{2}\right] \\
& =\mathfrak{q}_{4}^{n_{4}} \cdots \mathfrak{q}_{j+2}^{n_{j+2}} M+0_{\dot{M}}^{\dot{:}} x_{1}+x_{2}\left[\mathfrak{q}_{4}^{n_{4}} \cdots \mathfrak{q}_{j+2}^{n_{j+2}} M: x_{2}\right] .
\end{aligned}
$$

Here we used $\left(F_{j-1}\right)$. Thus $b \in \mathfrak{q}_{4}^{n_{4}} \cdots \mathfrak{q}_{j+2}^{n_{j+2}} M+0:_{M} x_{1}$ and $x_{2} a=x_{3} b+c \in$ $\mathfrak{q}_{3} \mathfrak{q}_{4}^{n_{4}} \cdots \mathfrak{q}_{j+2}^{n_{j+2}} M$

The proof of Lemma 7.1 is completed.

Lemma 7.2. With the same notation as in Lemma 7.1, we have

$$
\mathfrak{q}_{2}^{n_{2}} \cdots \mathfrak{q}_{j}^{n_{j}} M: x_{1}^{n_{1}}=\mathfrak{q}_{2}^{n_{2}} \cdots \mathfrak{q}_{j}^{n_{j}} M: \mathfrak{q}_{1}
$$

for any integers $2 \leq j \leq d, n_{1}>0$ and $n_{2}, \ldots, n_{j} \geq 0$.

Proof. If $n_{2}=\cdots=n_{j}=0$, then the equality is obvious. Therefore we may assume that one of $n_{2}, \ldots, n_{j}$ is positive. We may also assume that $n_{1}=1$ because of $\left(E_{j-1}\right)$. If $n_{2}=\cdots=n_{k-1}=0$ and $n_{k}>0$, then we obtain $\mathfrak{q}_{k}^{n_{k}} \cdots \mathfrak{q}_{j}^{n_{j}} M: x_{1} \subset$ $\mathfrak{q}_{k}^{n_{k}-1} \cdots \mathfrak{q}_{j}^{n_{j}} M+0:_{M} x_{1}$ by applying $\left(C_{j-k+1}\right)$ to the p-standard sequence $x_{1}, x_{k}$, $\ldots, x_{d}$ and hence

If $2 \leq i<k$, then we obtain

$$
\mathfrak{q}_{k}\left[\mathfrak{q}_{k}^{n_{k}} \cdots \mathfrak{q}_{j}^{n_{j}} M: x_{1}\right] \subset \mathfrak{q}_{k}^{n_{k}} \cdots \mathfrak{q}_{j}^{n_{j}} M
$$

$$
\begin{aligned}
\mathfrak{q}_{k}^{n_{k}} \cdots \mathfrak{q}_{j}^{n_{j}} M: x_{1} & \subset \mathfrak{q}_{k}^{n_{k}} \cdots \mathfrak{q}_{j}^{n_{j}} M: x_{1} x_{i} \\
& =\mathfrak{q}_{k}^{n_{k}} \cdots \mathfrak{q}_{j}^{n_{j}} M: x_{i}
\end{aligned}
$$


by applying $\left(F_{j-k+1}\right)$ to the p-standard sequence $x_{1}, x_{i}, x_{k}, \ldots, x_{d}$. Therefore

$$
\left(x_{1}, \ldots, x_{k-1}\right)\left[\mathfrak{q}_{k}^{n_{k}} \cdots \mathfrak{q}_{j}^{n_{j}} M: x_{1}\right] \subset \mathfrak{q}_{k}^{n_{k}} \cdots \mathfrak{q}_{j}^{n_{j}} M .
$$

Thus $\mathfrak{q}_{1}\left[\mathfrak{q}_{k}^{n_{k}} \cdots \mathfrak{q}_{j}^{n_{j}} M: x_{1}\right] \subset \mathfrak{q}_{k}^{n_{k}} \cdots \mathfrak{q}_{j}^{n_{j}} M$.

Lemma 7.3. With the same notation as Lemma 7.1, if $\mathfrak{q}_{2} M: x_{1}=\mathfrak{q}_{2} M$, then

$$
\left(x_{\lambda} \mid \lambda \in \Lambda\right) M: x_{1}=\left(x_{\lambda} \mid \lambda \in \Lambda\right) M
$$

for any subset $\Lambda \subset\{2, \ldots, d\}$. Furthermore

$$
\mathfrak{q}_{2}^{n_{2}} \cdots \mathfrak{q}_{j}^{n_{j}} M: x_{1}=\mathfrak{q}_{2}^{n_{2}} \cdots \mathfrak{q}_{j}^{n_{j}} M
$$

for any $1 \leq j<d$ and $n_{2}, \ldots, n_{j} \geq 0$.

Proof. We prove (7.3.1) by descending induction on the number of elements in $\Lambda$. If $\Lambda=\{2, \ldots, d\}$, then there is nothing to prove. Assume that $\Lambda \subsetneq\{2, \ldots, d\}$ and make the obvious inductive assumption. Let $j \in\{2, \ldots, d\} \backslash \Lambda$ and $a$ an element of the left hand side of (7.3.1). Then

$$
\begin{aligned}
a & \in\left[x_{j} M+\left(x_{\lambda} \mid \lambda \in \Lambda\right) M\right]: x_{1} \\
& =x_{j} M+\left(x_{\lambda} \mid \lambda \in \Lambda\right) M .
\end{aligned}
$$

Let $a=x_{j} b+c$ where $b \in M$ and $c \in\left(x_{\lambda} \mid \lambda \in \Lambda\right) M$. Then

$$
\begin{aligned}
b & \in\left(x_{\lambda} \mid \lambda \in \Lambda\right) M: x_{1} x_{j} \\
& =\left(x_{\lambda} \mid \lambda \in \Lambda\right) M: x_{j} .
\end{aligned}
$$

Therefore $a=x_{j} b+c \in\left(x_{\lambda} \mid \lambda \in \Lambda\right) M$.

Next we show (7.3.2). If $n_{2}=\cdots=n_{j}=0$, then the equation is trivial. We may assume that $n_{2}, n_{j}>0$. We work by induction on $j$. If $j=2$, then

$$
\mathfrak{q}_{2}^{n_{2}} M: x_{1}=\mathfrak{q}_{2}^{n_{2}-1}\left[\mathfrak{q}_{2} M: x_{1}\right]+0: x_{1}=\mathfrak{q}_{2}^{n_{2}} M
$$

Assume that $j>2$ and make the obvious inductive assumption. By using $\left(B_{j-1}\right)$, we may assume that $n_{2}=1$. Let $a$ be an element of the left hand side of (7.3.2). Since $x_{1}, x_{3}, \ldots, x_{d}$ is a p-standard sequence on $\bar{M}=M / x_{2} M$ and $\mathfrak{q}_{3} \bar{M}: x_{1}=$ $\mathfrak{q}_{3} \bar{M}$, we obtain that

$$
\begin{aligned}
a & \in\left[x_{2} M+\mathfrak{q}_{3}^{n_{3}+1} \cdots \mathfrak{q}_{j}^{n_{j}} M\right]: x_{1} \\
& =x_{2} M+\mathfrak{q}_{3}^{n_{3}+1} \cdots \mathfrak{q}_{j}^{n_{j}} M .
\end{aligned}
$$

Therefore

$$
\begin{aligned}
a & \in \mathfrak{q}_{3}^{n_{3}+1} \cdots \mathfrak{q}_{j}^{n_{j}} M+x_{2} M \cap\left[\mathfrak{q}_{2} \mathfrak{q}_{3}^{n_{3}} \cdots \mathfrak{q}_{j}^{n_{j}} M: x_{1}\right] \\
& =\mathfrak{q}_{3}^{n_{3}+1} \cdots \mathfrak{q}_{j}^{n_{j}} M+x_{2}\left[\mathfrak{q}_{3}^{n_{3}} \cdots \mathfrak{q}_{j}^{n_{j}} M: x_{1}\right] .
\end{aligned}
$$

Here we used $\left(D_{j-1}\right)$. By applying the inductive assumption to the p-standard sequence $x_{1}, x_{3}, \ldots, x_{d}$ on $M$, we have $\mathfrak{q}_{3}^{n_{3}} \cdots \mathfrak{q}_{j}^{n_{j}} M: x_{1}=\mathfrak{q}_{3}^{n_{3}} \cdots \mathfrak{q}_{j}^{n_{j}} M$. Therefore

$$
\begin{aligned}
a & \in \mathfrak{q}_{3}^{n_{3}+1} \cdots \mathfrak{q}_{j}^{n_{j}} M+x_{2} \mathfrak{q}_{3}^{n_{3}} \cdots \mathfrak{q}_{j}^{n_{j}} M \\
& =\mathfrak{q}_{2} \mathfrak{q}_{3}^{n_{3}} \cdots \mathfrak{q}_{j}^{n_{j}} M .
\end{aligned}
$$

The proof is completed. 


\section{Proofs of Theorems $1.2-1.4$}

We want to prove Theorems $1.2-1.4$ in this section. Let $A$ be a Noetherian ring and $\mathfrak{b}$ an ideal in $A$. The Rees algebra of $\mathfrak{b}$ is the graded ring

$$
R(\mathfrak{b})=A[\mathfrak{b} T]
$$

where $T$ is an indeterminate. We construct a Cohen-Macaulay Rees algebra.

First we consider the local case. Assume that $A$ is a Noetherian local ring and let $M$ be a finitely generated $A$-module of dimension $d>0$. We put $\mathfrak{a}^{\prime}(M)=$ $\prod_{p}$ ann $H^{p}\left(M^{\bullet}\right)$. It is well defined because $M^{p}=0$ if either $p<-1$ or $p>d$. The proof of the following lemma is quite similar to that of Theorem 3.3. The difference is only that we use Lemma 6.1 instead of Lemma 3.2.

Lemma 8.1. Let $x_{1}, \ldots, x_{d}$ be a system of parameters for $M$ and $1 \leq s<d$ an integer. If

$$
\left\{\begin{array}{l}
x_{s+1}, \ldots, x_{d} \in \mathfrak{a}^{\prime}(M), \\
x_{i} \in \mathfrak{a}^{\prime}\left(M /\left(x_{i+1}, \ldots, x_{d}\right) M\right), \quad \text { for } i \leq s,
\end{array}\right.
$$

then $x_{1}, \ldots, x_{d}$ is a p-standard sequence on $M$ of type $s$.

Proof. Replace " $\mathfrak{a}$ " and "Lemma 3.2" in the proof of Theorem 3.3 by " $\mathfrak{a}$ " and "Lemma 6.1", respectively.

Proposition 8.2. Let $A$ be a Noetherian local ring of dimension $d \geq 2, x_{1}, \ldots, x_{d}$ a system of parameters for $A$ and $0 \leq s<d-1$ an integer. We put $\mathfrak{q}_{i}=\left(x_{i}, \ldots, x_{d}\right)$ for all $1 \leq i \leq s+1$. If $x_{1}, \ldots, x_{d}$ is a p-standard sequence on $M$ of type $s$ and $0:{ }_{A} x_{d}=0$, then the Rees algebra $R\left(\mathfrak{q}_{1} \cdots \mathfrak{q}_{s} \mathfrak{q}_{s+1}^{d-s-1}\right)$ is a Cohen-Macaulay ring. If, in addition, $A / \mathfrak{q}_{t}$ is Cohen-Macaulay for some $1<t \leq s+1$, then $R\left(\mathfrak{q}_{t} \cdots \mathfrak{q}_{s} \mathfrak{q}_{s+1}^{d-s-1}\right)$ is Cohen-Macaulay.

Proof. Lemmas 7.1, 7.2 and 8.1 assure us that the sequence $x_{1}, \ldots, x_{d}$ satisfies (1)-(5) of Theorem 4.4 of [15]. Furthermore $0: x_{A}=0 \subset 0:_{A} x_{1}$. Thus the multi-Rees algebra

$$
A\left[\mathfrak{q}_{1} T_{1}, \ldots, \mathfrak{q}_{s} T_{s}, \mathfrak{q}_{s+1} T_{s+1}, \ldots, \mathfrak{q}_{s+1} T_{d-1}\right]
$$

is Cohen-Macaulay, where $T_{1}, \ldots, T_{d-1}$ are indeterminates, and hence the Rees algebra $R\left(\mathfrak{q}_{1} \cdots \mathfrak{q}_{s} \mathfrak{q}_{s+1}^{d-s-1}\right)$ is also. See [13, Corollary 2.10].

Next we assume that $A / \mathfrak{q}_{t}$ is Cohen-Macaulay for some $1<t \leq s+1$. That is, $x_{1}, \ldots, x_{t-1}$ is a regular sequence on $A / \mathfrak{q}_{t}$. We show that

$$
\left(x_{1}, \ldots, x_{i}\right): x_{d}=\left(x_{1}, \ldots, x_{i}\right) \text { for } 1 \leq i<t
$$

by induction on $i$. Assume that $i \geq 1$ and $\left(x_{1}, \ldots, x_{i-1}\right): x_{d}=\left(x_{1}, \ldots, x_{i-1}\right)$. Let $a \in\left(x_{1}, \ldots, x_{i}\right): x_{d}$ and put $x_{d} a=b+x_{i} c$ where $b \in\left(x_{1}, \ldots, x_{i-1}\right)$ and $c \in A$. Since $x_{i}, x_{t}, \ldots, x_{d}$ is a p-standard sequence on $A /\left(x_{1}, \ldots, x_{i-1}\right)$ and

$$
\left(x_{1}, \ldots, x_{i-1}, x_{t}, \ldots, x_{d}\right): x_{i}=\left(x_{1}, \ldots, x_{i-1}, x_{t}, \ldots, x_{d}\right),
$$

we obtain

$$
\begin{aligned}
c & \in\left(x_{1}, \ldots, x_{i-1}, x_{d}\right): x_{i} \\
& =\left(x_{1}, \ldots, x_{i-1}, x_{d}\right) .
\end{aligned}
$$


Here we used Lemma 7.3. Put $c=b^{\prime}+x_{d} a^{\prime}$ where $b^{\prime} \in\left(x_{1}, \ldots, x_{i-1}\right)$ and $a^{\prime} \in A$. Then

$$
\begin{aligned}
a-x_{i} a^{\prime} & \in\left(x_{1}, \ldots, x_{i-1}\right): x_{d} \\
& =\left(x_{1}, \ldots, x_{i-1}\right) .
\end{aligned}
$$

Therefore $a \in\left(x_{1}, \ldots, x_{i}\right)$.

Thus the sequence $x_{t}, \ldots, x_{d}$ on $\bar{A}=A /\left(x_{1}, \ldots, x_{t-1}\right)$ satisfies the assumption of Theorem 4.4 of [15]. Therefore the Rees algebra $R\left(\mathfrak{q}_{t} \cdots \mathfrak{q}_{s} \mathfrak{q}_{s+1}^{d-s-1} \bar{A}\right)$ is CohenMacaulay.

By using Lemma 7.3 and induction on $k$, we find that, if $1 \leq k \leq t-1$, then $x_{1}$, $\ldots, x_{k}$ is a regular sequence on $A$ and also on $A /\left(\mathfrak{q}_{t} \cdots \mathfrak{q}_{s} \mathfrak{q}_{s+1}^{d-\bar{s}-1}\right)^{n}$ for any $n>0$. We take the Koszul cohomologies of a short exact sequence

$$
0 \rightarrow R\left(\mathfrak{q}_{t} \cdots \mathfrak{q}_{s} \mathfrak{q}_{s+1}^{d-s-1}\right) \rightarrow A[T] \rightarrow \bigoplus_{n>0} A /\left(\mathfrak{q}_{t} \cdots \mathfrak{q}_{s} \mathfrak{q}_{s+1}^{d-s-1}\right)^{n} \rightarrow 0
$$

with respect to $x_{1}, \ldots, x_{t-1}$. Then we obtain that

$$
H^{p}\left(x_{1}, \ldots, x_{t-1} ; R\left(\mathfrak{q}_{t} \cdots \mathfrak{q}_{s} \mathfrak{q}_{s+1}^{d-s-1}\right)\right)=0 \quad \text { if } p<t-1
$$

and

$$
H^{t-1}\left(x_{1}, \ldots, x_{t-1} ; R\left(\mathfrak{q}_{t} \cdots \mathfrak{q}_{s} \mathfrak{q}_{s+1}^{d-s-1}\right)\right)=R\left(\mathfrak{q}_{t} \cdots \mathfrak{q}_{s} \mathfrak{q}_{s+1}^{d-s-1} \bar{A}\right) .
$$

Therefore $x_{1}, \ldots, x_{t-1}$ is a regular sequence on $R\left(\mathfrak{q}_{t} \cdots \mathfrak{q}_{s} \mathfrak{q}_{s+1}^{d-s-1}\right)$ and

$$
R\left(\mathfrak{q}_{t} \cdots \mathfrak{q}_{s} \mathfrak{q}_{s+1}^{d-s-1}\right) /\left(x_{1}, \ldots, x_{t-1}\right) R\left(\mathfrak{q}_{t} \cdots \mathfrak{q}_{s} \mathfrak{q}_{s+1}^{d-s-1}\right) \cong R\left(\mathfrak{q}_{t} \cdots \mathfrak{q}_{s} \mathfrak{q}_{s+1}^{d-s-1} \bar{A}\right)
$$

is Cohen-Macaulay. Hence $R\left(\mathfrak{q}_{t} \cdots \mathfrak{q}_{s} \mathfrak{q}_{s+1}^{d-s-1}\right)$ is Cohen-Macaulay.

Next we consider the non-local case.

Theorem 8.3. Let $A$ be a Noetherian catenary ring. Then the following statements are equivalent:

(1) A satisfies (C1) $-(\mathrm{C} 3)$;

(2) for every finitely generated $A$-module $M$ satisfying (QU), all the cohomology modules of the Cousin complex of $M$ are finitely generated and only finitely many of them are non-zero;

(3) for every ideal $I$ of $A$ such that $A / I$ satisfies (U) and $\operatorname{dim} A / I>0$, the ring $A / I$ has an arithmetic Macaulayfication.

Proof. $(1) \Rightarrow(2)$ : This implication is Theorem 1.1.

$(2) \Rightarrow(3)$ : It is enough to construct an arithmetic Macaulayfication of $A$ if $A$ satisfies (U) and $\operatorname{dim} A>0$.

If $\operatorname{dim} A=1$, then $A$ is Cohen-Macaulay because it has no embedded primes. There is a non-zero divisor $a$ which is not a unit. Then the Rees algebra $R(a A)$ is isomorphic to a polynomial ring over a Cohen-Macaulay ring $A$ and hence is Cohen-Macaulay.

Assume that $\operatorname{dim} A \geq 2$, including the case that $\operatorname{dim} A=\infty$. We want to choose integers $q \geq p \geq 2$ and elements $x_{1}, \ldots, x_{q}$ in $A$ such that

$$
\left\{\begin{aligned}
\operatorname{ht}\left(x_{1}, \ldots, x_{k}\right) & =k \quad \text { for any } k>0, \\
x_{1}, \ldots, x_{p} & \in \mathfrak{a}^{\prime}(A), \\
x_{k+1} & \in \mathfrak{a}^{\prime}\left(A /\left(x_{1}, \ldots, x_{k}\right)\right) \quad \text { if } k \geq p
\end{aligned}\right.
$$


and $A /\left(x_{1}, \ldots, x_{q}\right)$ is Cohen-Macaulay, including the case that $\operatorname{dim} A /\left(x_{1}, \ldots, x_{q}\right)=$ 0 . By applying (2) to $A$, we find that $\mathfrak{a}^{\prime}(A)$ is well defined and $V\left(\mathfrak{a}^{\prime}(A)\right)$ is equal to the non-Cohen-Macaulay locus of $A$, as in the proof of Corollary 6.4. Since $A$ has no embedded primes, we obtain $V\left(\mathfrak{a}^{\prime}(A)\right) \subset U^{2}(A)$. Let $p=\operatorname{ht} \mathfrak{a}^{\prime}(A)$. Then $p \geq 2$ and there are $x_{1}, \ldots, x_{p} \in \mathfrak{a}^{\prime}(A)$ such that ht $\left(x_{1}, \ldots, x_{k}\right)=k$ for any $k \leq p$. Assume that there are elements $x_{1}, \ldots, x_{q^{\prime}}$ in $A$ satisfying (8.3.1) and $p \leq q^{\prime}$. Since $A$ is catenary and satisfies (U), $A /\left(x_{1}, \ldots, x_{q^{\prime}}\right)$ satisfies (QU). Therefore $\mathfrak{a}^{\prime}\left(A /\left(x_{1}, \ldots, x_{q^{\prime}}\right)\right)$ is well defined. If $A /\left(x_{1}, \ldots, x_{q^{\prime}}\right)$ is not Cohen-Macaulay, then there is $x_{q^{\prime}+1} \in \mathfrak{a}^{\prime}\left(A /\left(x_{1}, \ldots, x_{q^{\prime}}\right)\right)$ such that $\mathrm{ht}\left(x_{1}, \ldots, x_{q^{\prime}+1}\right) /\left(x_{1}, \ldots, x_{q^{\prime}}\right)=1$. In this case, $\operatorname{ht}\left(x_{1}, \ldots, x_{q^{\prime}+1}\right)=q^{\prime}+1$ because $A$ satisfies (U). This procedure must stop after a finite number of iterations because $A$ is Noetherian. Thus we obtain a sequence satisfying (8.3.1) and such that $A /\left(x_{1}, \ldots, x_{q}\right)$ is Cohen-Macaulay.

Let $\mathfrak{b}=\left(x_{1}, \ldots, x_{q}\right) \cdots\left(x_{1}, \ldots, x_{p+1}\right)\left(x_{1}, \ldots, x_{p}\right)^{p-1}$. Then ht $\mathfrak{b}>0$ because $x_{1}^{q-1} \in \mathfrak{b}$ and ht $x_{1} A=1$. We show that the Rees algebra $R=R(\mathfrak{b})$ is CohenMacaulay. Let $\mathfrak{P}$ be a prime ideal of $R$ and $\mathfrak{p}=\mathfrak{P} \cap A$.

If $\left(x_{1}, \ldots, x_{p}\right) \not \subset \mathfrak{p}$, then $A_{\mathfrak{p}}$ is Cohen-Macaulay and $\mathfrak{b} A_{\mathfrak{p}}=A_{\mathfrak{p}}$. Therefore $R_{\mathfrak{p}}$ is a polynomial ring over a Cohen-Macaulay ring and hence $R_{\mathfrak{P}}$ is Cohen-Macaulay.

If $x_{1}, \ldots, x_{k} \in \mathfrak{p}$ but $x_{k+1} \notin \mathfrak{p}$ for some $k \geq p$, then $x_{1}, \ldots, x_{k}$ is a subsystem of parameters for $A_{\mathfrak{p}}$ because $A$ satisfies (U). If $\operatorname{dim} A_{\mathfrak{p}} /\left(x_{1}, \ldots, x_{k}\right) A_{\mathfrak{p}}>0$, then we take $y_{1}, \ldots, y_{l} \in \mathfrak{p}$ such that $y_{1}, \ldots, y_{l}, x_{1}, \ldots, x_{k}$ form a system of parameters for $A_{\mathfrak{p}}$. Since the construction of Cousin complexes commutes with localization, the system of parameters $y_{1}, \ldots, y_{l}, x_{k}, \ldots, x_{1}$ for $A_{\mathfrak{p}}$ satisfies (8.1.1) and $A_{\mathfrak{p}} /\left(x_{k}, \ldots, x_{1}\right) A_{\mathfrak{p}}$ is Cohen-Macaulay. Since

$$
\mathfrak{b} A_{\mathfrak{p}}=\left(x_{k}, \ldots, x_{1}\right) \cdots\left(x_{p+1}, \ldots, x_{1}\right)\left(x_{p}, \ldots, x_{1}\right)^{p-1} A_{\mathfrak{p}},
$$

we find that $R_{\mathfrak{p}}=R\left(\mathfrak{b} A_{\mathfrak{p}}\right)$ is Cohen-Macaulay. If $\operatorname{dim} A_{\mathfrak{p}} /\left(x_{1}, \ldots, x_{k}\right) A_{\mathfrak{p}}=0$, then $x_{k}, \ldots, x_{1}$ form a system of parameters for $A_{\mathfrak{p}}$ satisfying (8.1.1). Therefore $R_{\mathfrak{p}}$ is Cohen-Macaulay.

If $x_{1}, \ldots, x_{q} \in \mathfrak{p}$, then we can also show that $R_{\mathfrak{p}}$ is Cohen-Macaulay in the same way as above.

$(3) \Rightarrow(1)$ : Let $\mathfrak{p}$ be a prime ideal. If $\operatorname{dim} A / \mathfrak{p} \leq 1$, then $A / \mathfrak{p}$ is Cohen-Macaulay. If $\operatorname{dim} A / \mathfrak{p} \geq 2$, then $A / \mathfrak{p}$ has an arithmetic Macaulayfication and hence $A / \mathfrak{p}$ is a homomorphic image of a Cohen-Macaulay ring. In any case $A / \mathfrak{p}$ satisfies (C1), (C2) and hence $A$ also does.

Next we consider (C3). Let $B$ be a finitely generated $A$-algebra, $\mathfrak{P}$ a prime ideal of $B$ and $\mathfrak{p}=\mathfrak{P} \cap A$. We want to show that the Cohen-Macaulay locus of $B / \mathfrak{P}$ contains a non-empty open subset of $\operatorname{Spec} B / \mathfrak{P}$. Since $A / \mathfrak{p}$ is a homomorphic image of a Cohen-Macaulay ring, there is an element $x \in A \backslash \mathfrak{p}$ such that $(A / \mathfrak{p})_{x}$ is Cohen-Macaulay. Since $B / \mathfrak{P}$ is a finitely generated $A / \mathfrak{p}$-algebra, $(B / \mathfrak{P})_{x}$ is a homomorphic image of a Cohen-Macaulay ring. Therefore the Cohen-Macaulay locus of $(B / \mathfrak{P})_{x}$ contains a non-empty open subset of $\operatorname{Spec}(B / \mathfrak{P})_{x}$, and hence the Cohen-Macaulay locus of $B / \mathfrak{P}$ contains a non-empty open subset of Spec $B / \mathfrak{P}$.

The proof of Theorem 8.3 is completed.

We prove Theorem 1.2. The implication $(2) \Rightarrow(1)$ is contained in Theorem 8.3. We want to prove the opposite implication. If $A$ has an arithmetic Macaulayfication, then it is a homomorphic image of a Cohen-Macaulay ring. Therefore $A$ satisfies (C1)-(C3). We prove that $A$ satisfies (U) if $A$ has an arithmetic Macaulayfication $R=R(\mathfrak{b})$. Let $\mathfrak{p}$ be an associated prime of $A$. Assume that ht $\mathfrak{p}>0$. If $\mathfrak{b} \not \subset \mathfrak{p}$, then 
a polynomial ring $R_{\mathfrak{p}}$ over $A_{\mathfrak{p}}$ is Cohen-Macaulay. Hence $A_{\mathfrak{p}}$ is a Cohen-Macaulay ring. If $\mathfrak{b} \subset \mathfrak{p}$, then $A_{\mathfrak{p}}$ has an arithmetic Macaulayfication $R_{\mathfrak{p}}$ and hence $A_{\mathfrak{p}}$ is unmixed [15, Theorem 1.1]. In any case, $A_{\mathfrak{p}}$ is unmixed and $\operatorname{dim} A_{\mathfrak{p}}>0$. This contradicts the fact that $\mathfrak{p}$ is an associated prime of $A$. Thus $A$ has no embedded primes.

Let $\mathfrak{p}, \mathfrak{q}$ be prime ideals in $A$ such that $\mathfrak{p} \subset \mathfrak{q}$. In the same way as above, we find that $A_{\mathfrak{q}}$ is unmixed and hence ht $\mathfrak{p} A_{\mathfrak{q}}+\mathrm{ht} \mathfrak{q} / \mathfrak{p}=\mathrm{ht} \mathfrak{q} A_{\mathfrak{q}}$. The proof of Theorem 1.2 is completed.

Theorem 1.3 comes from Theorem 1.2. If $A$ satisfies $(\mathrm{C} 1)-(\mathrm{C} 3)$ and has a codimension function, then $A$ is a homomorphic image of a finitely generated $A$ algebra $B$ with codimension function $t$ such that $t$ has a constant value $t_{0}$ on all the associated primes of $B$ [15, Lemma 5.5]. Then $B$ has no embedded primes. Since ht $(-)=t(-)-t_{0}$ is a codimension function, $B$ satisfies (U). It is obvious that $B$ satisfies (C1) and (C3). Furthermore, we can show that $B$ satisfies (C2) by modifying the proof of [23, Theorem 5.7]. Thus $B$ has an arithmetic Macaulayfication, and hence $A$ is a homomorphic image of a Cohen-Macaulay ring. The opposite implication is obvious.

Theorem 1.4 is contained in Theorem 8.3.

Finally we mention that the existence of a codimension function is not superfluous in Theorem 1.3. Ogoma [16, 5.I] gave an example of a Noetherian ring $A$ which is universally catenary, that is, satisfies $(\mathrm{C} 1)$, but has no codimension function. We note that $A$ also satisfies (C2) and (C3). His example has exactly two maximal ideals $\mathfrak{m}_{1}, \mathfrak{m}_{2}$ and exactly two minimal primes $\mathfrak{p}_{1}, \mathfrak{p}_{2}$ such that ht $\mathfrak{m}_{1} / \mathfrak{p}_{1}=$ ht $\mathfrak{m}_{2} / \mathfrak{p}_{1}=$ ht $\mathfrak{m}_{1} / \mathfrak{p}_{2}=2$, ht $\mathfrak{m}_{2} / \mathfrak{p}_{2}=1, V\left(\mathfrak{p}_{1}\right) \cap V\left(\mathfrak{p}_{2}\right)=\left\{\mathfrak{m}_{1}, \mathfrak{m}_{2}\right\}$ and $A / \mathfrak{p}_{1}, A / \mathfrak{p}_{2}$ are regular. Since $A / \mathfrak{p}_{1}, A / \mathfrak{p}_{2}$ are regular, $A$ satisfies (C2). If $\mathfrak{p}$ is a prime ideal in $A$, then $A / \mathfrak{p}$ is a regular ring, a 1-dimensional domain or a field and hence Cohen-Macaulay. Therefore we find that $A$ satisfies (C3).

\section{REFERENCES}

[1] Yôichi Aoyama, Some basic results on canonical modules, J. Math. Kyoto Univ. 23 (1983), no. 1, 85-94. MR692731 (84i:13015)

[2] Nguyễn Tư' Cu'ò'ng, On the length of the powers of systems of parameters in local ring, Nagoya Math. J. 120 (1990), 77-88. MR1086571 (92b:13033)

[3] - On the dimension of the non-Cohen-Macaulay locus of local rings admitting dualizing complexes, Math. Proc. Cambridge Philos. Soc. 109 (1991), no. 3, 479-488. MR1094747 (92b:13034)

[4] M. T. Dibaei and M. Tousi, The structure of dualizing complex for a ring which is $\left(\mathrm{S}_{2}\right)$, J. Math. Kyoto Univ. 38 (1998), no. 3, 503-516. MR1661220 (99k:13020)

[5] - A generalization of the dualizing complex structure and its applications, J. Pure Appl. Algebra 155 (2001), no. 1, 17-28. MR1804325 (2001m:13022)

[6] Gerd Faltings, Der Endlichkeitssatz in der lokalen Kohomologie, Math. Ann. 255 (1981), no. 1, 45-56. MR611272 (82f:13003)

[7] Robert Fossum, Hans-Bjørn Foxby, Phillip Griffith, and Idun Reiten, Minimal injective resolutions with applications to dualizing modules and Gorenstein modules, Inst. Hautes Études Sci. Publ. Math. (1975), no. 45, 193-215. MR0396529 (53:392)

[8] Shiro Goto, Approximately Cohen-Macaulay rings, J. Algebra 76 (1982), no. 1, 214-225. MR659220 (84h:13033)

[9] Shiro Goto and Kikumichi Yamagishi, The theory of unconditioned strong d-sequences and modules of finite local cohomology, preprint.

[10] Janet E. Hall, Fundamental dualizing complexes for commutative Noetherian rings, Quart. J. Math. Oxford Ser. (2) 30 (1979), no. 117, 21-32. MR528888 (80h:13016) 
[11] Robin Hartshorne, Residues and duality, Lecture notes of a seminar on the work of A. Grothendieck, given at Harvard 1963/64. With an appendix by P. Deligne. Lecture Notes in Mathematics, No. 20, Springer-Verlag, Berlin, 1966. MR0222093 (36:5145)

[12] Craig Huneke, The theory of d-sequences and powers of ideals, Adv. in Math. 46 (1982), no. 3, 249-279. MR683201 (84g:13021)

[13] Eero Hyry, The diagonal subring and the Cohen-Macaulay property of a multigraded ring, Trans. Amer. Math. Soc. 351 (1999), no. 6, 2213-2232. MR1467469 (99i:13005)

[14] Takesi Kawasaki, On Macaulayfication of Noetherian schemes, Trans. Amer. Math. Soc. 352 (2000), no. 6, 2517-2552. MR1707481 (2000j:14077)

[15] On arithmetic Macaulayfication of Noetherian rings, Trans. Amer. Math. Soc. 354 (2002), no. 1, 123-149 (electronic). MR1859029 (2002i:13001)

[16] Tetsushi Ogoma, Fibre products of Noetherian rings and their applications, Math. Proc. Cambridge Philos. Soc. 97 (1985), no. 2, 231-241. MR771818 (86e:13008)

[17] Henrike Petzl, Cousin complexes and flat ring extensions, Comm. Algebra 25 (1997), no. 1, 311-339. MR1429765 (98c:13024)

[18] L. J. Ratliff, Jr., Characterizations of catenary rings, Amer. J. Math. 93 (1971), 1070-1108. MR0297752 (45:6804)

[19] Peter Schenzel, Cohomological annihilators, Math. Proc. Cambridge Philos. Soc. 91 (1982), no. 3, 345-350. MR654081 (83j:13008)

[20] Standard systems of parameters and their blowing-up rings, J. Reine Angew. Math. 344 (1983), 201-220. MR716256 (84m:13025)

[21] R. Y. Sharp, Cousin complex characterizations of two classes of commutative Noetherian rings, J. London Math. Soc. (2) 3 (1971), 621-624. MR0294323 (45:3392)

[22] Rodney Y. Sharp, The Cousin complex for a module over a commutative Noetherian ring., Math. Z. 112 (1969), 340-356. MR0263800 (41:8400)

[23] _ Acceptable rings and homomorphic images of Gorenstein rings, J. Algebra 44 (1977), no. 1, 246-261. MR0441957 (56:348)

[24] Local cohomology and the Cousin complex for a commutative Noetherian ring, Math. Z. 153 (1977), no. 1, 19-22. MR0442062 (56:450)

[25] Ngô Viêt Trung, Toward a theory of generalized Cohen-Macaulay modules, Nagoya Math. J. 102 (1986), 1-49. MR846128 (87h:13018)

Department of Mathematics, Tokyo Metropolitan University, Hachioji-shi Minami OHSAWA 1-1,D TOKYO, 192-0397, JAPAN

E-mail address: kawasaki@tmu.ac.jp $U R L:$ http://www.comp.metro-u.ac.jp/ kawasaki/ 